\title{
Future High Precision Experiments and New Physics Beyond Standard Model
}

\author{
Mingxing Luo
}

\author{
Institute for Nuclear Theory, HN-12, and Department of Physics, FM-15, \\ University of Washington, Seattle, WA 98195
}

$\mathrm{DOE} / \mathrm{ER} / 40561--095$

DE93 011004

\section{DISCLAIMER}

\begin{abstract}
This report was prepared as an account of work sponsored by an agency of the United States Government. Neither the United States Government nor any agency thereof, nor any of their employees, makes any warranty, express or implied, or assumes any legal liability or responsibility for the accuracy, completeness, or usefulness of any information, apparatus, product, or process disclosed, or represents that its use would not infringe privately owned rights. Reference herein to any specific commercial product, process, or service by trade name, trademark, manufacturer, or otherwise does not necessarily constitute or imply its endorsement, recommendation, or favoring by the United States Government or any agency thereof. The views and opinions of authors expressed herein do not necessarily state or reflect those of the United States Government or any agency thereof.
\end{abstract}

\section{PREPARED FOR THE U.S. DEPARTMENT OF ENERGY UNDER GRANT DE-FG06-90ER40561}

This report was prepared as an account of work sponsored by the United States Government. Neither the United States nor any agency thereof, nor any of their employees, makes any warranty, express or implied, or assumes any legal liability or responsibility for the accuracy, completeness, or usefulness of any information, apparatus, product, or process disclosed, or represents that its use would not infringe privately owned rights. Reference herein to any specific commercial product, process, or service by trade name, mark, manufacturer, or otherwise, does not necessarily constitute or imply its endorsement, recommendation, or favoring by the United States Government or any agency thereof. The views and opinions of authors expressed herein do not necessarily state or reflect those of the United States Government or any agency thereof. 
Future High Precision Experiments

and New Physics Beyond Standard Model

\author{
Mingxing Luo \\ Institute for Nuclear Theory, University of Washington \\ Seattle, WA 98195, USA
}

\begin{abstract}
This paper analyzes of the high precision $(\leq 1 \%)$ electroweak experiments that have been done or are likely to be done in this decade. Starting with the Standard Model (SM) predictions of fourteen weak neutral current observables and fifteen $W$ and $Z$ properties to the one-loop level, we investigated the implications of the corresponding experimental measurements to various types of possible new physics that enter at the tree or loop level. Certain experiments appear to have special promise as probes of the new physics considered here.
\end{abstract}

\title{
Contents
}

1 Introduction 1

2 Formalism 5

3 Physical Observables and Their Measurements 8

4 New Physics 11

4.1 Extra $Z$ bosons........................ 11

4.2 Extra scalar bosons . . . . . . . . . . . . . . . . . 12

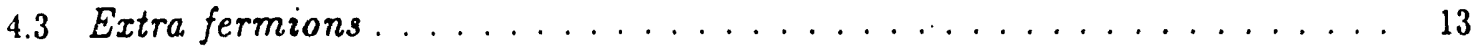

4.4 Contact operators . . . . . . . . . . . . . . . . . . . 13

4.5 Heavy particle loop contributions .................... 13

5 Comparison of experiments vs new physics 14

6 Conclusions $\quad 19$

\section{Introduction}

A few years ago, a theoretical framework was developed for the analysis of the high precision electroweak experiments that were likely to be done in the next ten years [1]. Since then, significant experimental progresses have been made, especially at the $e^{+} e^{-}$collider LEP at CERN [2]. The mass of the $Z$ boson $M_{Z}$ has been shifted from 91.177 to $91.187 \mathrm{GeV}$, the error on $M_{Z}, \Delta M_{Z}$, has been reduced from 0.031 to $0.007 \mathrm{GeV}$, which is much better than the error anticipated. The errors on the various $Z$ decay widths have also been improved to be better than anticipated. Furthermore, there 
are preliminary results of the various asymmetries at the $Z$ pole in the $e^{+} e^{-}$collision. The error on forward-backward asymmetry of $\mu$ is again better than anticipated. On the other hand, the global analysis of existing $W$ and $Z$ particle properties and weak-neutral current data [3] yields a new constraint on the mass of the top quark, $m_{t}=150_{-23-25}^{+17+15}$. We update the analysis by incorporating these new experimental results. We will choose the value of $M_{Z}=91.187 \pm 0.007 \mathrm{GeV}, m_{t}=150 \mathrm{GeV}$, and $M_{H}=250 \mathrm{GeV}$ as inputs for the theoretical prediction, in contrast with the inputs $M_{Z}=91.177 \pm 0.021 \mathrm{GeV}, m_{t}=100 \mathrm{GeV}$, and $M_{H}=100 \mathrm{GeV}$ used in [1].

Historically, the electroweak experiments played an important role in the establishment of the the Standard Model (SM). The new experiments considered in this paper, each designed to have a precision of the order of one percent or better, will test the electroweak interaction component of the SM, the Glashow-WeinbergSalam theory [4], to high precision, and might possibly detect new physics beyond the SM. They provide a complementary alternative to the direct method of studying higher energy phenomena to find departures from the SM.

The establishment of the SM is one of the major accomplishments in particle physics during the past twenty years. The SM is mathematically self-consistent and is compatible with all known experimental data [5]. But there are many theoretical questions that cannot be answered satisfactorily within the framework of the SM. For example, the SM has a complicated gauge structure and many free parameters.

If we believe the unification of all the fundamental forces and the selfdetermination of physical parameters within the theory, we have to seek a more fundamental theory, which is not subject to these shortcomings but reduces to the $\mathrm{SM}$ at present energies. To do so, we construct new theories and models, such as grand unified theories, supersymmetry, and superstrings. Experimentally, we try to detect new physical phenomena which might be induced by such underlying new physics. One way is to build higher energy colliders. There is, however, a complementary approach. That is, to improve the precision of present electroweak experiments. If there exists physics beyond the SM, there might be remnants of it at present energies which would cause small deviations of physical quantities from the SM values. These deviations would be too small to be observed given the present experimental errors, but might become amenable to more precise experiments.

In this decade, there have been and will be performed a series of high precision experiments relating to the properties of the $Z$ - and $W$-bosons and to weak neutral current observables. As mentioned at the beginning, the $Z$-boson mass has already been measured very precisely [2]. The decaying widths of $z$, the forwardbackward and possibly polarization asymmetries in $e^{+} e^{-}$collisions, and the $W$ boson mass have been or will be measured with high but somewhat less precision (see [6][9] and references in [1]). High precision measurements of neutrino-electron scattering, atomic parity violation, deep inelastic neutrino scattering, and other neutral current processes are all likely to be performed (see [10]-[13] and references in [1]). In Table 1.1, we have collected the relevant observables, their expectations in the SM, and their present and projected experimental uncertainties.

For the analysis of high precision experiments, we have to take another impor- 
Table 1.1: The observables considered in this article, their SM predictions, and their present and future experimental uncertainties (including theoretical uncertainties where they are important). The SM predictions use the observed value of $M_{Z}$ and assume $m_{t}=150 \mathrm{GeV}$, and $M_{H}=250 \mathrm{GeV}$. $g_{L}^{2}, g_{R}^{2}, R_{\nu}, \theta_{L}$, and $\theta_{R}$ are quantities measured in $\nu N$ scattering; $g_{V}^{e}, g_{A}^{e}, \sigma_{\nu} / \sigma_{\nu}$, and $\sigma_{\nu} /\left(\sigma_{\nu}+\sigma_{\nu}\right)$ are releyant to $\nu e$ scattering; $C_{1 \pm}, C_{2 p m}$ are measured in atomic parity violation, muonic atoms, and $I N$ scattering; $A_{L R}, A_{F B}$, and $A_{p o l}$ are asymmetries at the $Z$-pole, and the $\Gamma$ 's are the partial and total $Z$ widths.

\begin{tabular}{|c|c|c|c|c|c|c|}
\hline \multirow{2}{*}{$\begin{array}{c}\text { Quantities } \\
O_{a}\end{array}$} & \multirow[b]{2}{*}{$O_{a}^{S M}$} & \multicolumn{3}{|c|}{ (present) } & \multicolumn{2}{|c|}{ (future) } \\
\hline & & $O_{a}^{\exp }$ & $\Delta O_{a}^{\exp }$ & $\Delta \sin ^{2} \theta_{w}^{\text {ezp }}$ & $\Delta O_{a}^{\text {exp }}$ & $\Delta \sin ^{2} \theta_{w}^{\exp }$ \\
\hline$\overline{M_{Z}(\mathrm{GeV})}$ & $=$ & 91.187 & 0.007 & 0.0003 & $=$ & $\bar{z}$ \\
\hline$M_{W}(\mathrm{GeV})$ & 80.267 & 80.1 & 0.3 & 0.0018 & 0.105 & 0.0006 \\
\hline$g_{L}^{2}$ & 0.3025 & 0.3003 & 0.0039 & 0.0052 & - & - \\
\hline$g_{R}^{2}$ & 0.030 & 0.0323 & 0.0033 & 0.013 & - & - \\
\hline$R_{v}$ & 0.315 & - & - & - & 0.001 & 0.002 \\
\hline$\theta_{L}$ & 2.46 & 2.49 & 0.035 & - & - & - \\
\hline$\theta_{R}$ & 5.18 & 4.58 & 0.41 & - & - & - \\
\hline$g_{V}^{e}$ & -0.039 & -0.035 & 0.017 & 0.009 & - & - \\
\hline$g_{A}^{e}$ & -0.505 & -0.508 & 0.015 & - & - & 一 \\
\hline$\sigma_{\nu} / \sigma_{\bar{\nu}}$ & 1.164 & 1.083 & 0.10 & 0.012 & 0.046 & 0.005 \\
\hline$\sigma_{\nu} /\left(\sigma_{\bar{\nu}}+\sigma_{\nu}\right)$ & 0.148 & - & - & 一 & 0.0027 & 0.0025 \\
\hline$C_{1+}$ & 0.129 & 0.126 & 0.003 & 0.01 & 0.0013 & 0.003 \\
\hline$C_{1+}($ iso $)$ & 0.129 & - & $\longrightarrow$ & - & 0.0003 & 0.0009 \\
\hline$C_{1-}$ & -0.367 & -0.45 & 0.1 & 0.07 & - & - \\
\hline$C_{2 p}$ & -0.015 & - & - & - & 0.046 & - \\
\hline$C_{2 p}(1)$ & -0.015 & - & - & - & 0.0046 & 一 \\
\hline$C_{2 m}$ & -0.059 & - & - & - & 0.11 & 0.03 \\
\hline $2 C_{1 u}+C_{16}$ & -0.036 & - & - & 一 & 0.004 & 0.002 \\
\hline$A_{L R}(S L C)$ & 0.140 & - & - & - & 0.0066 & 0.0008 \\
\hline$A_{L R}(L E P)$ & 0.140 & - & - & - & 0.0041 & 0.0005 \\
\hline$A_{F B}^{p o l}(c)$ & 0.476 & - & - & - & 0.025 & 0.01 \\
\hline$A_{F B}(c)$ & 0.067 & 0.072 & 0.027 & 0.0065 & 0.007 & 0.0017 \\
\hline$A_{F B}^{p o l}(b)$ & 0.697 & - & - & - & 0.02 & 0.04 \\
\hline$A_{F B}(b)$ & 0.098 & 0.098 & 0.012 & 0.002 & 0.0054 & 0.001 \\
\hline$A_{F B}^{p o l}(\mu)$ & 0.105 & - & 一 & - & 0.009 & 0.0015 \\
\hline$A_{F B}(\mu)$ & 0.015 & 0.0174 & 0.0027 & 0.0017 & 一 & - \\
\hline$A_{p o l}(\tau)$ & 0.140 & 0.140 & 0.018 & 0.0025 & 0.01 & 0.0014 \\
\hline$\Gamma_{\text {inv }}(\mathrm{GeV})$ & 0.501 & 0.505 & 0.006 & 0.0025 & - & - \\
\hline$\Gamma_{1 i}(\mathrm{GeV})$ & 0.0838 & 0.0833 & 0.0003 & 0.0006 & - & - \\
\hline$\Gamma_{\epsilon \bar{c}}(\mathrm{GeV})$ & 0.298 & 0.235 & 0.038 & 0.02 & 0.03 & 0.016 \\
\hline$\Gamma_{b \bar{b}}(\mathrm{GeV})$ & 0.376 & 0.373 & 0.009 & 0.004 & - & - \\
\hline$\Gamma_{Z}(\mathrm{GeV})$ & 2.493 & 2.492 & 0.007 & 0.0005 & - & - \\
\hline
\end{tabular}


Table 1.2: The types of new physics considered in this paper

\begin{tabular}{ll}
\hline Tree level physics: & extra $Z: \chi, \psi, \eta, Z_{L R}$ \\
& non-standard Higgs representations \\
& leptoquarks: type I, type II \\
& extra fermions: $u_{L, R}^{\prime}, d_{L, R}^{\prime}, e_{L, R}^{\prime}, \nu_{L, R}^{\prime}$ \\
& compositeness: four-fermi contact operators \\
\hline \hline Loop level physics: & $m_{t}, M_{H}$ \\
& extra fermions \\
& $S-T$ parameters: gauge boson self-energies \\
& two Higgs doublets \\
& supersymmetry \\
\hline
\end{tabular}

tant physical phenomenon into serious consideration, namely radiative corrections within the SM (see [14], [15], and references in [1]). The radiative corrections are generally small with a few exceptions; they are usually in the order of one percent of the Born approximation values. (The radiative corrections to the masses of $W$ and $Z$ bosons are about 4 percent.) With one percent experimental errors, one has to include the radiative corrections for physical observables in the analysis. They are essential to the verification of the SM. Furthermore, they must be included if one is to search for small deviations due to new physics. Radiative corrections at the oneloop level to all of the SM predictions have been included in our analysis. Note that the SM is now established as correct to an excellent first approximation. Hence, we can apply the SM radiative corrections, treating any new physics (whether it enters at tree or loop level) as a perturbation.

In [1], we investigated ten general types of possible new physics that enter at the tree or loop level, as listed in Table 1.2. For new physics entering at the tree level, we considered extra $Z$ bosons, extra scalar bosons, extra fermions, and compositeness. For new physics entering at the loop level, we considered heavy extra fermions which break the custodial symmetry, extra Higgs doublets, S-T parameters, and supersymmetry. The unknown top quark and Higgs boson masses, $m_{t}$ and $M_{H}$, enter predictions of the observables through radiative corrections. We include these in Table 1.2 even though they are not technically "new physics", because the techniques for constraining them are similar to those in the search for new physics and because they lead to complications in the analysis. We also took 27 specific examples to illustrate our formalism [1]. Five of these examples will be updated in this paper, other cases can be updated in a similar manner. A brief summary of the implications to all the 27 specific examples will also be included.

In our formalism we emphasize the interplay of experimental possibilities and theoretical models. We explore how experimental data will constrain theories and how theories may guide future experiments. The testing of the SM and searching for new physics require the comparison of a number of precise experiments. Most observables are not absolute predictions of the SM, but depend on $\sin ^{2} \theta_{W}$. A single 
experimental result can usually be accommodated by choosing $\sin ^{2} \theta_{W}$ appropriately. However, comparison of the values of $\sin ^{2} \theta_{W}$ obtained by a number of experiments probes for physics beyond the SM. If a series of high precision experiments yield equal values for $\sin ^{2} \theta_{w}$ (within experimental and theoretical uncertainties) then the SM is successfully tested at that level and limits can be set on the possible contributions of new physics.

On the other hand, observed deviations would be evidence for new physics. In this case one wants as much information as possible to distinguish with clarity the different kinds of new physics. We describe two schemes to distinguish one type of new physics from another which serve as a prescription for the analysis of future high precision experiments. One possibility is to use the $\sin ^{2} \theta_{W}$ values extracted from the different experiments. The pattern of deviations of the various $\sin ^{2} \theta_{W}$ from each other may be a distinguishing feature. Another and more powerful possibility is to compare the observables directly with the SM predictions. Again, the pattern of deviations is a diagnostic of the type of new physics. In either case, the most sensitive probe for new physics involves the totality of experiments. The high precision electroweak experiments and our theoretical framework comprise one method to probe incisively and exhaustively for any weakness in the SM, and to recognize and identify it if found.

Following this introductory section, we provide a general formalism for the analysis in Section 2; Section 3 is a survey of high precision experiments; Section 4 is a general discussion of various types of possible new physics; in Section 5 are comparison of experiments and possible new physics; in Section 6 are the conclusions.

\section{Formalism}

Consider a physical observable $O_{a}$ and denote its experimental value as $O_{a}^{\exp }$ and the theoretical prediction as $O_{a}^{T}$. Then. within experimental and theoretical uncertainties,

$$
O_{a}^{T}=O_{a}^{e x p}
$$

if both theory and experiment are correct (the superscript ${ }^{T}$ will be omitted in the following for convenience). Starting from the SM as our best guess, we have the SM prediction $O_{a}^{S M}$ of $O_{a}$. Were the SM the true theory, then

$$
O_{a} \equiv O_{a}^{S M}=O_{a}^{e x p}
$$

Were there new physics beyond the SM, then

$$
O_{a}=O_{a}^{S M}+\sum_{i} \Delta O_{a}^{i}
$$

where $\Delta O_{a}^{i}$ is the contribution to $O_{a}$ of the new physics $i$. We have assumed that contributions from new physics are sufficiently small compared with that from the 
SM that they can be calculated perturbatively and contributions from different new physics can be simply summed up.

In general, $O_{a}^{S M}$ depends on the $S U(2) \times U(1)$ gauge structure and the free parameters of the theory. In the GWS theory, besides the fermion masses, there are three free parameters, namely:

$$
\alpha, G_{F}, \sin ^{2} \theta_{W},
$$

the QED coupling constant, the Fermi weak interaction coupling constant, and the weak mixing angle. $\alpha$ and $G_{F}$ have already been determined precisely by other experiments. $\sin ^{2} \theta_{W}$ is the single unspecified parameter in the GWS theory, representing the mixing between the $S U(2)$ and $U(1)$ sectors. At tree level, $\sin ^{2} \theta_{W}$ is given by $g^{\prime 2} /\left(g^{\prime 2}+g^{2}\right)$, where $g$ and $g^{\prime}$ are respectively the $S U(2)$ and $U(1)$ gauge coupling constants. To go beyond the tree level, one must define a renormalized $\sin ^{2} \theta_{W}$. One possibility is the on-shell definition $\sin ^{2} \theta_{W}^{M}=1-M_{W}^{2} / M_{Z}^{2}$, where $M_{W}$ and $M_{Z}$ are the measured masses. Another possibility is the $\overline{M S}$ definition $\sin ^{2} \hat{\theta}_{W}\left(M_{Z}\right)$. The two definitions are related by $\sin ^{2} \hat{\theta}_{W}\left(M_{Z}\right)=C\left(m_{t}, M_{H}\right) \sin ^{2} \theta_{W}^{M}$, where $C=1.029$ for $m_{t}=150 \mathrm{GeV}, M_{H}=250 \mathrm{GeV}$. These definitions should yield the same predictions if some appropriate summations of high order terms are taken ${ }^{2}$. However, since we are dealing with deviations from the SM to the lowest order of perturbation, our analysis is essentially independent of which definition of $\sin ^{2} \theta_{W}$ is used.

Express both $O_{a}$ and $O_{a}^{S M}$ as functions of $\sin ^{2} \theta_{W}$,

$$
O_{a}=O_{a}\left(\sin ^{2} \theta_{W}\right), \text { and } O_{a}^{S M}=O_{a}^{S M}\left(\sin ^{2} \theta_{W}\right) .
$$

Since we are ignorant of the exact value of $\sin ^{2} \theta_{W}$, we define an effective $\sin ^{2} \theta_{W}^{a}$ for each $O_{a}$ by assuming the validity of the SM:

$$
O_{a}\left(\sin ^{2} \theta_{W}\right)=O_{a}^{S M}\left(\sin ^{2} \theta_{W}^{a}\right)
$$

with

$$
\sin ^{2} \theta_{W}^{a}=\sin ^{2} \theta_{W}+\Delta \sin ^{2} \theta_{W}^{a} .
$$

where $\Delta \sin ^{2} \theta_{W}^{a}$ is the shift away from the true $\sin ^{2} \theta_{W}$ due to new physics. From (3), (5), and (6) we have

$$
\Delta \sin ^{2} \theta_{W}^{a}=\frac{\sum_{i} \Delta O_{a}^{i}}{\left.\frac{d O_{d}^{S M}}{d x}\right|_{x^{*}}}
$$

where $x^{a}=\sin ^{2} \theta_{W}^{a}$. The validity of the SM requires that all $\Delta \sin ^{2} \theta_{W}^{a}$ should vanish, and all of the $\sin ^{2} \theta_{W}^{a}$ should be equal to $\sin ^{2} \theta_{W}$ (within uncertainties). The $\sin ^{2} \theta_{W}^{a}$ would differ from one another if there is new physics beyond the SM. Thus, one way to test the SM is simply to compare the values of $\sin ^{2} \theta_{w}^{a}$ extracted from measurements of differerit physical quantities $O_{a}$.

However, not all physical quantities are sensitive to $\sin ^{2} \theta_{W}$. For example, the axial part of $\iota^{-e} e$ couplings is essentially independent of $\sin ^{2} \theta_{W}$. So when using the $\sin ^{2} \theta_{W}$ analysis, it is possible to trade a relatively precise experiment for a poor

\footnotetext{
${ }^{1}$ For a thorough discussion of $\sin ^{2} \theta_{W}$, see $\{1\}$ and references within.
} 
result. It is important to have predictions of the physical quantities themselves. We therefore consider two analyses: one in which the $O_{a}$ are compared directly and one in which the extracted $\sin ^{2} \theta_{W}^{a}$ are compared. In the former no information is lost, while the latter is easier to use. To study the $O_{a}$, we need a numerical estimate of $\sin ^{2} \theta_{w}$ to compute the SM prediction. There are two reasonable choices for the estimate of $\sin ^{2} \theta_{W}$ : one is the world average value [3] $\sin ^{2} \theta_{W}^{a v g}=0.2260 \pm 0.0024$ (onshell) or $\sin ^{2} \theta_{W}^{a v g}=0.2325 \pm 0.0007(\overline{M S})$; another possibility is $\sin ^{2} \theta_{W}^{Z}$ extracted from $M_{Z}$. Both $\sin ^{2} \theta_{W}^{a v g}$ and $\sin ^{2} \theta_{W}^{Z}$ may be shifted from the true $\sin ^{2} \theta_{W}$ by new physics, and that shift must be taken into account in the analysis. This is easier to do for $\sin ^{2} \theta_{W}^{Z}$, since it comes from a single measured quantity, and $\sin ^{2} \theta_{W}^{Z}$ is used in this paper.

Expressing the equations above in terms of $\sin ^{2} \theta_{W}^{Z}$, we have

$$
\sin ^{2} \theta_{W}^{a}=\sin ^{2} \theta_{W}^{Z}+\Delta \sin ^{2} \theta_{W}^{(a ; Z)}
$$

where

$$
\Delta \sin ^{2} \theta_{W}^{(a) Z)}=\Delta \sin ^{2} \theta_{W}^{a}-\Delta \sin ^{2} \theta_{W}^{Z}
$$

i.e. $\Delta \sin ^{2} \theta_{W}^{(a ; Z)}$ represents both the shift in $\sin ^{2} \theta_{W}^{a}$ and in. $\sin ^{2} \theta_{W}^{Z}$ due to new physics. A nonzero $\Delta \sin ^{2} \theta_{w}^{(a, Z)}$ (beyond uncertainties) would indicate new physics. Eqs. (8), (9), and (10) are the basic results for the $\sin ^{2} \theta_{W}$ analysis. The value of $\Delta \sin ^{2} \theta_{W}^{(a ; z)}$ obtained from experiments by the differences between the measured and the SM values shown in (9) can be compared with the predictions from (8) and (10) for each type of new physics. For the analysis based directly on the observables $O_{a}\left(\sin ^{2} \theta_{W}\right)$ one uses:

$$
\begin{aligned}
O_{a}\left(\sin ^{2} \theta_{W}\right) & =O_{a}^{S M}\left(\sin ^{2} \theta_{W}\right)+\sum_{i} \Delta O_{a}^{i} . \\
& =O_{a}^{S M}\left(\sin ^{2} \theta_{W}^{Z}\right)-\left.\frac{d O_{a}^{S M}}{d x}\right|_{x} z \Delta \sin ^{2} \theta_{W}^{Z}+\sum_{i} \Delta O_{a}^{i} \\
& =O_{a}^{S M}\left(\sin ^{2} \theta_{W}^{Z}\right)+\Delta O_{a}
\end{aligned}
$$

We will refer to the SM prcdiction as:

$$
O_{a}^{S M}\left(\sin ^{2} \theta_{W}^{Z}\right)
$$

while the contribution from the new physics is

$$
\Delta O_{a}=-\left.\frac{d O_{a}^{S M}}{d x}\right|_{x} z \Delta \sin ^{2} \theta_{W}^{z}+\sum_{i} \Delta O_{a}^{i} .
$$

At the one-loop level, $\Delta O_{a}$ is independent of the renormalization scheme. Again, the first term in $\Delta O_{a}$ represents the difference between the (assumed) value of $\sin ^{2} \theta_{W}^{Z}$ and the true $\sin ^{2} \theta_{w}$, while the second term is the direct effect of the new physics on $O_{a}$. The calculated $O_{a}\left(\sin ^{2} \theta_{W}\right)$ in (11) are the quantities to be directly compared with experiment. The experimental values of $\Delta O_{a}$ obtained from (11) can therefore be compared with the predictions for each type of new physics calculated by $(7)$ to (13). 


\section{Physical Observables and Their Measurements}

In this section we discuss the physical observables. It is summarized in Table 1.1 which gives the SM predictions $O_{a}^{S M}\left(\sin ^{2} \theta_{W}^{Z}\right)$ for twenty-nine observables and the present and anticipated experimental and theoretical errors $(1 \sigma)$. For details and references, please see [6]-[13], [1] and references within.

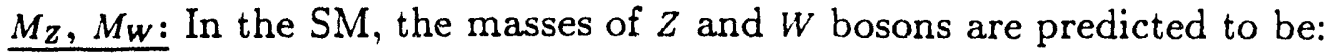

$$
M_{Z}^{2}=\frac{\pi \alpha / \sqrt{2} G_{F}}{\rho \cos ^{2} \theta_{W} \sin ^{2} \theta_{W}(1-\Delta r)}, M_{W}^{2}=\frac{\pi \alpha / \sqrt{2} G_{F}}{\sin ^{2} \theta_{W}(1-\Delta r)},
$$

to the one-loop level; where $\Delta r$ and $\rho-1$ are radiative correction parameters which have been calculated and which depend on and the renormalization scheme and $m_{t}$, $M_{H}$. The LEP experiments yield the extremely precise value $M_{Z}=91.187 \pm 0.007 \mathrm{GeV}$. This value of $M_{Z}$ together with the assumed values $m_{t}=150 \mathrm{GeV}$ and $M_{H}=250 \mathrm{GeV}$ are used as the input parameters in the numerical calculations that follow, and are referred to as the standard input parameters (SIP) in the remainder of the paper. With these inputs $\sin ^{2} \theta_{W}^{Z}=0.2259 \pm 0.0003$ (on-sinell) or $0.2325 \pm 0.0003(\overline{M S}$ ). The present world-averaged value of $M_{W}$ is $80.1 \pm 0.3 \mathrm{GeV}$, which leads to an error of 0.0018 in $\sin ^{2} \theta_{W}$ for the SIP, independent of renormalization scheme. One expects improved measurements by three independent techniques: $W$ production at the Tevatron, $W$ exchange at HERA, and the reaction $e^{+} e^{-}-W^{+} W^{-}$in the second phase of LEP. (LEP 200). A reasonable projection of the experimental error on $M_{W}$ is $100 \mathrm{MeV}$, which would lead to an error of 0.0006 in $\sin ^{2} \theta_{w}$.

Low energy effective neutrino-quark coupling coefficients: The low energy effective Lagrangian for neutrino-quark interaction can be written as:

$$
-L_{e f f}=\frac{G_{F}}{\sqrt{2}} \sum_{\alpha=1}^{n} \bar{\nu} \gamma^{\mu}\left(1-\gamma_{5}\right) \nu \sum_{i}\left[\epsilon_{L}(i) \bar{q}_{i} \gamma_{\mu}\left(1-\gamma_{5}\right) q_{i}+\epsilon_{R}(i) \bar{q}_{i} \gamma_{\mu}\left(1+\gamma_{5}\right) q_{i}\right]
$$

The explicit expressions of the $\epsilon$ 's in SM are given in [1]. The quantities extracted from experiments are the following combinations of the $\epsilon_{L, R}: g_{L}^{2}=\epsilon_{L}^{2}(u)+\epsilon_{L}^{2}(d)$, $g_{R}^{2}=\epsilon_{R}^{2}(u)+\epsilon_{R}^{2}(d), \theta_{L}=\tan ^{-1} \epsilon_{L}(u) / \epsilon_{L}(d), \theta_{R}=\tan ^{-1} \epsilon_{R}(u) / \epsilon_{R}(d)$. Their SM predictions and experimental status are listed in Table 1.1. Measurements of $\epsilon_{L, R}$, especially the measurements of the deep inelastic scattering cross-section ratios $R_{\nu}=\sigma\left(\nu_{\mu} N \rightarrow\right.$ $\left.\nu_{\mu} X\right) / \sigma\left(\nu_{\mu} N \rightarrow \mu^{-} X\right)$ and $R_{\bar{\nu}}=\sigma\left(\bar{\nu}_{\mu} N \rightarrow \bar{\nu}_{\mu} X\right) / \sigma\left(\bar{\nu}_{\mu} N \rightarrow \mu^{+} X\right)$ on approximately isoscalar targets are significant accomplishments of the second and the third generation weak neutral current experiments. In the future, there may be deep inelastic experiments using a high energy $\nu_{\mu}$ beam, e.g., at Fermilab. At high energies the theoretical uncertainties will be reduced and one may obtain a measurement of $R_{\nu} \simeq g_{L}^{2}+0.4 g_{R}^{2}$ corresponding to $\Delta \sin ^{2} \theta_{w}=0.002$ or better.

Low energy effective $\nu_{\mu}$-electron coupling coefficients: Similar to the $\nu-q$ interaction, one has the effective Lagrangian of the $\nu_{1}$-electron interaction:

$$
-L_{e j \rho}^{\nu_{\mu} e}=\frac{G_{F}}{\sqrt{2}} \overline{\nu_{\mu}} \gamma^{\mu}\left(1-\gamma_{5}\right) \nu_{\mu} \bar{e} \gamma_{\mu}\left(g_{V}^{e}-g_{A}^{e} \gamma_{5}\right) e,
$$


(see [1] for the SM predictions of $g_{V, A}^{e}$ ) Their SM predictions and present experimental values are listed in Table 1.1. The measurement of the ratio $g_{V} / g_{A}$ is expected to be improved, but not the separate values. Here we emphasis two types of experiments which minimize possible systematic errors and which, with sufficient statistics, would yield precise values of the measured quantities. One is of the ratio: $R_{\nu / \bar{\nu}}=\sigma_{\nu_{\mu} e \rightarrow \nu_{\mu} e} / \sigma_{\bar{\nu}_{\mu} e \rightarrow \bar{\nu}_{\mu} e}$ to be performed at CERN. Another is of the ratio: $R_{\nu / \bar{\nu} \nu_{e}}=\sigma_{\nu_{\mu} e \rightarrow \nu_{\mu} e} /\left(\sigma_{\bar{\nu}_{\mu} e \rightarrow \bar{\nu}_{\mu} e}+\sigma_{\nu_{e} e-\nu_{e} e}\right)$ proposed at Los Alamos.

Atomic parity violation coupling constants: In general, the parity violating coupling between electron and hadron at low energy can be parameterized as follows:

$$
-L^{e H}=-\frac{G_{F}}{\sqrt{2}} \sum_{i}\left(C_{1 i} \bar{e} \gamma_{\mu} \gamma_{5} e \overline{q_{i}} \gamma^{\mu} q_{i}+C_{2 i} \bar{e} \gamma_{\mu} e \overline{q_{i}} \gamma^{\mu} \gamma_{5} q_{i}\right)
$$

(see [1] for the SM predictions of $C$ 's.) There are various ways to measure $C$ 's. We consider the following combinations which are the quantities extracted from experiments: $C_{1+}=0.666 C_{1 u}+0.747 C_{1 d}, C_{1-}=0.747 C_{1 u}-0.666 C_{1 d}, C_{2 p}=C_{2 u}+C_{2 d}$ and $C_{2 m}=C_{2 u}-C_{2 d}$, where the comvination $C_{1+}$ corresponds to parity-violation in the cesium atom. Their SM predictions and experimental statues are listed in Table 1.1. The present experimental values of the coefficients are: $C_{1+}=0.126 \pm 0.003$ and $C_{1-}=-0.45 \pm 0.10 ; C_{2 p}$ and $C_{2 m}$ are poorly determined. The experimental error of $C_{1}+$ may soon be reduced to $<0.5 \%$. The total error would then be dominated by the theoretical uncertainty of $\sim 1 \%$ [16]. Future measurements involving different isotopes of cesium or other elements will determine the $C_{1}$ 's more precisely [17]. We project two uncertainties for $C_{1+}:$ a $1 \%$ uncertainty expected soon $\left(C_{1+}\right)$ and a later possible $0.2 \%$ uncertainty from the use of different isotopes $\left(C_{1+}(\right.$ iso $\left.)\right)$.

Asymmetries of $e^{+} e^{-}$scattering at the $Z$-pole: given by:

a) The left-right asymmetry with longitudinally-polarized initial electrons is

$$
A_{L R}=\frac{\sigma\left(e_{L}^{-} e^{+}-\sum_{f} f \bar{f}\right)-\sigma\left(e_{R}^{-} e^{+} \rightarrow \sum_{f} f \bar{f}\right)}{\sigma\left(e_{\bar{L}}^{-} e^{+}-\sum_{f} f \bar{f}\right)+\sigma\left(e_{\bar{R}}^{-} e^{+} \rightarrow \sum_{f} f \bar{f}\right)},
$$

where the summation runs through all fermion pairs $f \vec{f}$ comprising the final states. $A_{L R}$ is small but sensitive to $\sin ^{2} \theta_{W}$, so its precise measurement will provide an excellent determination of $\sin ^{2} \theta_{W}$. In particular, the extracted $\sin ^{2} \hat{\theta}_{W}\left(M_{Z}\right)$ is insensitive to $m_{\mathfrak{t}}$ and $h_{H}$. Within the SIP, $A_{L R}=0.140$. The projected experimental error of $A_{L R}$ at LEP is 0.003 if LEP goes ahead with polarization, corresponding to an error of 0.0003 in $\sin ^{2} \theta_{W}$. At SLC, an error in $A_{L R}$ of 0.006 is expected. However, we must fold in the theoretical error $\Delta A_{L R}^{\text {pred }} \simeq 0.003$ in the SM prediction of $A_{L R}$, from $\Delta M_{Z}$ and the uncertainty in $\Delta r$. The effective error is therefore $\Delta A_{L R} \simeq 0.004$ (LEP) or $0.006 b$ (SLC), corresponding to $\Delta \sin ^{2} \theta_{W} \simeq 0.0005$ (0.0008).

b) The forward-backward asymmetries of final state fermions are:

$$
A_{F B}(f)=\frac{\left(\int_{\cos \theta>0}-\int_{\cos \theta<0}\right) d \Omega \frac{d \sigma}{d \Omega}\left(e^{+} e^{-}-\bar{f} f\right)}{\left(\int_{\cos \theta>0}+\int_{\cos \theta<0}\right) d \Omega \frac{d \sigma}{d \Omega}\left(e^{+} e^{-}-\tilde{f} f\right)} .
$$

with $f=u, d, \mu$, etc. Here $\theta$ is the angle between the directions of the incoming electron and outgoing fermion $f$. The tree level predictions for various $A_{F B}$ 's are 
given in [1]. Their SM predictions and experimental errors are listed in Table 1.1. There are already some preliminary results of them from LEP [2]. We note that the errors on the forward-backward asymmetry of the $\mu$ is 0.0027 , which is lower than the anticipated error 0.0035 .

c) The polarized-forward-backward asymmetry is:

$$
A_{F B}^{p o l}(f)=\frac{\sigma_{F B}^{L}(f)-\sigma_{F B}^{R}(f)}{2 \sigma}
$$

where

$$
\begin{gathered}
\sigma_{F B}^{L, R}(f)=\left[\int_{\cos \theta>0}-\int_{\cos \theta<0}\right] d \Omega \frac{d \sigma}{d \Omega}\left(e_{\bar{L}, R}^{-} e^{+}-f \bar{f}\right), \\
\sigma=\int d \Omega \frac{d \sigma}{d \Omega}\left(e^{-} e^{+} \rightarrow f \bar{f}\right) .
\end{gathered}
$$

The tree level predictions for $f=u, d, \mu$ are included in [1], and the SM predictions and experimental errors in Table 1.1. There are still no experimental results yet.

d) Final state polarization asymmetries of the $r$ lepton: Define:

$$
A_{p o l}(\tau)=\frac{\sigma\left(e^{-} e^{+}-\tau_{L}^{-} \tau^{+}\right)-\sigma\left(e^{-} e^{+} \rightarrow \tau_{R}^{-} \tau^{+}\right)}{\sigma\left(e^{-} e^{+}-\tau_{L}^{-} \tau^{+}\right)+\sigma\left(e^{-} e^{+}-\tau_{R}^{-} \tau^{+}\right)}
$$

In the SM, it measures the $V$ and $A$ interference. The preliminary result of $A_{p o l}(\tau)$ is $0.140 \pm 0.018$. The error $\Delta A_{\text {por }}(\tau)$ will be improved to be 0.01 .

$Z$ decay widths:

a) Partial $Z$-decay widths: the $Z$ decays into lepton and quark pairs with different widths:

$$
\Gamma_{f f} ; f=\nu, e, u, d, \ldots
$$

Their tree level SM expressions are listed in [1]. And their SM predictions and experimental status are listed in Table 1.1. The experimental leptonic width is the averaged width of $e, \mu$ and $\tau$; we denote it as $\Gamma_{1 i}$. The width into unobserved (invisible) particles, $\Gamma_{i n v}$ is determined by the difference of the total $Z$-width (see below) and the $Z$-width into observable particles (hadrons and charged particles). The errors on most of the partial widths are more precisely than anticipated. Currently, $\Delta \Gamma_{l i}=0.0003 \mathrm{GeV}, \Delta \Gamma_{\text {inv }}=0.006 \mathrm{GeV}, \Delta \Gamma_{b \bar{b}}=0.009 \mathrm{GeV}$.

b) Inclusive $Z$-decay width: The total or inclusive $Z$-decay width is the summation of all the partial widths of fermions. That is:

$$
\Gamma_{z}=3 \Gamma_{z \rightarrow \nu \dot{\nu}}+3 \Gamma_{z-e+e-}+2 \Gamma_{z \rightarrow u u ̈}+3 \Gamma_{z-d \bar{d}},
$$

where the coefficients are due to the 3 families (the $t$ is too massive to contribute). This quantity is measured by the shape of the energy dependence of the total crosssection of the $e^{+{ }_{p}-}$ collision around the $Z$-pole. With the SIP, $\Gamma_{Z}=2.493 \mathrm{GeV}$. Experimentally, $\Gamma_{z}=2.492 \pm 0.00 \tau \mathrm{GeV}$. The errors is significantly lower than the anticipated $0.015 \mathrm{GeV}$. 


\section{New Physics}

In this section we briefly review the new physics considered in our analysis. For the details, please see [1] and references within.

\subsection{Extra $Z$ bosons}

Extra intermediate vector bosons (IVBs) beside the SM prediction of the $W$ and $Z$ arise naturally in Grand Unification Theories (GUTs) and other extensions of the SM, due to their larger gauge groups. These IVBs are usually too heavy to be relevant to physics in the laboratory since their mass are related to the large mass scale of GUTs and other theories. However, there can be extra IVBs whose masses are independent of the large mass scale. And they may gain masses by other Higgs fields at low energy, e.g., in the $T e V$ range. In that case, we may either see them directly in colliders, or find their effects at present energies in high precision experiments. Here we are interested the extra neutral $Z$ bosons. For a theory with the $S M$ as its first order approximation plus $n-1$ extra $Z$ bosons, the gauge group is $S U(2) \times \prod_{\alpha=1}^{n} U(1)_{\alpha}$. This symmetry is broken, in the process the IVBs acquire masses and there results the mixing among them. The mixing is caused by the diagonalization of the gauge boson mass matrix. These extra IVBs in general open a new channel for the weak neutral current processes, and their mixing with the ordinary $Z$ changes the coupling of fermions with the ordinary $Z$. The mixing also changes the mass of the ordinary $Z$. Since $\sin ^{2} \theta_{W}^{Z}$ derived from $M_{Z}$ is used as input in our analysis, the change of $M_{Z}$ shifts the SM predictions for physical quanitities.

To be concrete and to follow the spirit of grand unification, we take the $U(1)$ 's as relics of an underlying non-abelian gauge group, in which there is only one overall coupling constant and the fermion charges in one irreducible representation are related. The strength depends on the GUT breaking pattern. In this paper we take the $E(6)$ model as an important example. We also consider the extra $Z$ boson in $S U(2)_{L} \times S U(2)_{R} \times U(1)$ models. For these models, there are effectively two extra free parameters in the SM with an extra $Z$ boson,

$$
\begin{aligned}
& \hat{\rho}_{2}=\left(\frac{g_{2}}{g_{1}}\right)^{2}\left(\frac{M_{Z}}{M_{Z_{2}}}\right)^{2} \\
& \hat{\Theta}=\frac{g_{2}}{g_{1}} \Theta,
\end{aligned}
$$

where $\|_{Z, Z_{2}}$ and $g_{1,2}$ are the masses and coupling constants of the ordinary $Z$ and the extra $Z_{2}$, respectively; $\Theta$ is the $Z_{1}^{0}-Z_{2}^{0}$ mixing angle. Typically, $\left(g_{2} / g_{1}\right)^{2} \simeq 5 \sin ^{2} \theta_{W} / 3 \simeq$ 0.38. Both $\dot{\rho}_{2}$ and $\dot{\Theta}$ are small numbers, but their ratio $C=\dot{\Theta} / \hat{\rho}_{2}$ is of the order of unity. $C$ depends on the $U(1)_{2}$ quantum numbers of the Higgs fields which cause the mixing. In our analysis. $\dot{\rho}_{2}$ is taken to be the free parameter $\lambda$ and some typical values of $C$ (usually motivated by simple $E(6)$ models) are selected for each type of $Z_{2}$. 


\subsection{Extra scalar bosons}

It is always possible to incorporate scalar bosons into gauge theories without spoiling the symmetry. In the minimal SM, one $S U(2)$ scalar doublet employed to realize the Figgs mechanism. After spontaneous symmetry breaking, one scalar particle remains. $S U(3)$ is not broken in QCD, so no colored scalar bosons are introduced. In theories beyond the SM, extra scalar bosons usually cannot be avoided. In Grand Unification Theories, more scalar bosons must be introduced to break the symmetries via the Higgs mechanism. In technicolor theories there are no elementary scalars but scalars are generated as bound states. In supersymmetry, two Higgs dout,iets are lequired, and for each ordinary fermion there is a scalar partner. Scalars may have either color or weak charges. In this section, we take the non-standard Higgs fields as an example for color-singlet scalars and leptoquarks ior color-non-singlet scalars.

\section{A. Non-standarc: Higgs boson}

In the SM, $\rho_{0}=M_{W}^{2} / M_{Z}^{2} \cos ^{2} \theta_{W}$ is unity. In theories beyond the SM, if (colorsinglet) scalars which carry non-standard $S U(2)$ charges (i.e., $S U(2)$ triplets) develop vacuum expectation values, $\rho_{0}$ will be different from unity. The non-standard Higgs fields thus manifest themselves at tree level. Additional Higgs doublets do not change the SM prediction $\rho_{0}=1$. The shift of $\rho_{0}$ changes the prediction of $M_{2}$ and quantities related to it are directly affected (except for negligible effects associated with scalar exchange). However, $\sin ^{2} \theta_{W}^{Z}$ extracted from $M_{Z}$ assuming the validity of the SM differs from the true $\sin ^{2} \theta_{W}$. This gives artificial changes in quantities which are sensitive to $\sin ^{2} \theta_{W}$ when they are predicted from $\sin ^{2} \theta_{W}^{Z}$.

\section{B. Leptoquarks}

For colored bosons we consider the leptoquarks. They are color triplets which change quarks to leptons (or antileptons) and vice versa when they are emitted or absorbed. They have many experimental consequences, but we only consider effects on weak neutral current phenomena. The contributions of leptoquarks are suppressed at the $Z$ pole, as are those of photons; since their contributions are $\pi / 2$ out of the phase at the $Z$-pole. Accordingly, $e^{+} e^{-}$collider experiments at the $Z$-pole will give no information about them. Neither will leptoquarks contribute to pure leptonic processes; since leptoquarks change quarks to leptons and leptons to quarks at tree level. Only the $\nu q$ coupling constants and atomic parity-violation coefficients can be affected by leptoquarks.

In a general leptoquark theory, there may be many extra parameters. To be concrete, we take the following simple example for illustration: those which are predicted in the $S U(5)$ unification theory. In this example, there are only two relevant parameters: $\sqrt{2}\left|\eta_{L}\right|^{2} / 8 M_{S}^{2} G_{F}$ and $\sqrt{2}\left|\eta_{R}\right|^{2} / 8 M_{S}^{2} G_{F}$, where $\eta_{L, R}$ are coupling strengths. To be more specific, we take two definite examples: type-1 leptoquark: with $\eta_{L} \neq 0$ and $\eta_{R}=0 ;$ type-2 leptoquark: with $\eta_{L}=0$ and $\eta_{R} \neq 0$. 


\subsection{Extra fermions}

Most extensions of the SM predict the existence of extra fermions [18]. For instance, in the $E(6)$ model fermions in one family are assigned to a 27-plet, which includes the 15 ordinary fermions and 12 extra ones. Among the 12 extra fermions, we have one vector-singlet $D$ quark, $D_{L}, D_{R}$; one vector-doublet lepton: $\left(\begin{array}{c}E^{0} \\ E^{-}\end{array}\right)_{L},\left(\begin{array}{c}E^{0} \\ E^{-}\end{array}\right)_{R}$; and two Weyl neutrinos $\bar{N}_{L}, S_{L}^{0}$. Here we will not consider the actual production of new heavy fermions. Rather, we will focus on their mixing with the known light particles. This can affect weak universality, lead to induced right-handed currents, and affects the relations between the Fermi-constant and $M_{W, z}$. Specifically, we consider the mixing between ordinary fermions and the exotic right-handed $S U(2)$ doublets and left-handed $S U(2)$ singlets, which modifies the neutral current couplings of the light particles.

The theories of extra fermions are complicated by many free parameters. To simplify, we separate a general theory with many extra fermions into specific ones with only one extra fermion. So we do not deal with a theory with $n$ extra fermions, but $n$ theories with one extra fermion. In each theory, we have a single free parameter, $\sin ^{2} \theta$, where $\theta$ is the mixing angle between the ordinary and exotic fermions.

\subsection{Contact operators}

No hint has been seen of lepton or quark form factors or other signs of compositeness. Nevertheless, it is possible that the leptons and quarks have a substructure if the compositeness scale $\Lambda$ is very large $(\Lambda>O(1 \mathrm{TeV}))$. One consequence should be the generation by constituent interchange of four-fermi or other effective operators at energies small compared to $\Lambda$. These must be $S U(3) \times S U(2) \times U(1)$ invariant since new physics at the scale $\Lambda \gg M_{Z}$ must preserve the low-energy symmetries. One can write a great many invariant operators including those which generate $\mathrm{FCNC} ;, P$, and $T$ operators; and those which contribute to both charged and neutral current phenomena. Even restricting to $V, A$ operators which affect WNC processes only, there are many possibilities. We consider three representative cases of four-fermi contact operators: $\bar{l}_{\mu L} \gamma^{\mu} I_{\mu L} \bar{q}_{L} \gamma_{\mu} q_{L}$, which will shift the values of $\epsilon_{L}(u), \epsilon_{L}(d), C_{2 u}$, and $C_{2 d} ; \bar{\nu}_{\mu L} \gamma^{\mu} \nu_{\mu L} \bar{e}_{L} \gamma_{\mu} e_{L}$ which will shift the values of $g_{V}^{e}$ and $g_{A}^{e} ; \bar{e}_{L} \gamma^{\mu} e_{L} \bar{q}_{L} \gamma_{\mu} q_{L}$ which will shift the values of $C_{1 u}$ and $C_{1 d}$.

\subsection{Heavy particle loop contributions}

In this section we will discuss those which only affect the neutral current and $W$ and $Z$ observables through radiative corrections. As usual, these types of new physics are related to a high mass scale since they are not observed at the present energy level. Most types of heavy physics affect low energy observables only through inverse powers of the the heavy scale and therefore have little effect except possibly for 
mediating rare processes. However, that is not true if the heavy physics breaks the symmetries of the low energy theory. These heavy physics will affect the neutral current and $W, Z$ observables by shifting the self-energy diagrams. These shifts can be parametrized in terms of three parameters, $h_{V}=T, h_{A W}$ and $h_{A Z}$, where $h_{V}$ $\left(h_{A}\right)$ refers to the breaking of the vector (axial) part of $S U(2)$. In most cases, the axial parameters are approximately equal, $h_{A W} \sim h_{A Z}=S . h_{V}$ is sensitive to nondegenerate $S U(2)$ multiplets which break the vector $S U(2)$ symmetries, including the top quark mass, non-degenerate fourth family fermions, non-degenerate Higgs multiplets, and $\tilde{b}-\tilde{t}$ splitting in supersymmetry. The effects of $h_{V}$ are equivalent to $\rho_{0} \neq 1$. It is a very powerful parameterization, many types of new physics which enter at the one-loop level affect only the gauge boson self-energies and can be described by the parameters $h_{V}, h_{A W}$, and $h_{A Z}$. Among them, the important examples are: theories with extra fermion multiplets, the theories with two Higgs doublets, and the minimal supersymmetric standard model (MSSM).

In this paper, $M_{H}=250 \mathrm{GeV}$ and $m_{t}=150 \mathrm{GeV}$ are used as inputs for the calculation of radiative corrections. The effects of other values of $m_{t}$ and $M_{H}$ are treated as if they are new physics. Most of the effects of a Higgs mass $M_{H}$ or top quark mass $m_{l}$ differing from their reference values can be described by $h_{V}$ and $h_{A}$. However, they are treated separately in order to take the correlations between $h_{V}$, $h_{A W}$, and $h_{A Z}$ properly into account and to include additional $m_{t}$ dependence from $Z \rightarrow \bar{b} b$ vertex diagrams. The radiative corrections to physical observables depend logarithmatically on $M_{H}$ and are generally small. The effects of different values of $m_{t}$ are considerably larger due to the dominant $\alpha m_{t}^{2} / M_{Z}^{2}$ dependence of the ratio $M_{W}^{2} / M_{Z}^{2}$ and of quantities that depend on the ratio.

\section{Comparison of experiments vs new physics}

In Table 1.1, we have collected the measurements and the projected experimental errors of the observables $O_{a}, \Delta O_{a}^{\exp }$ and $\Delta \sin ^{2} \theta_{W}^{e x p}$, and the SM predictions of the observables $O_{a}$ up to one-loop radiative corrections. The effects of a variety of possible types of new physics involving extra $Z$ bosons, extra scalar bosons, extra fermions, compositeness, and types of new physics which enter at the loop level were discussed in last section. For the formulas of $\Delta O_{a}^{i}$ and $\Delta \sin ^{2} \theta_{W}^{a}$, please see [1].

This section compares the experiments with new physics. We will take five of the 27 examples considered in [1] for illustration. The results are collected in Tables 5.1 to 5.5 and the accompanying figures. The SM predictions for the quantities in column 1 are given in column 2. Column 3 gives the projected one standard deviation experimental errors, $\Delta O_{a}^{\text {exp }}$, which include the theoretical uncertainties when they are significant. The latter include both the theoretical uncertainties in the extraction of $O_{G}^{e x p}$ and the uncertainties in the SM prediction of $O_{a}$ due to the uncertainty $\sim 0.0003$ in $\sin ^{2} \theta_{W}^{Z}$. The contributions of new physics of type $i$ to each of the observables, $\Delta O_{a}^{i}$, is given in column 4 . To ensure accuracy, in each case 
the $\Delta O_{a}^{i}$ were determined by two independent calculations. The $\Delta O_{a}^{i}$ depend on a coupling constant ${ }^{2}, \lambda$, which determines the strength of the new physics. We assume $\lambda=0.01$ for definiteness in column 4 , but $\lambda$ can be scaled linearly to any value preferred by the reader. In column 5 is the minimum value of $\lambda$ for a given observable; $\lambda_{a}^{\min }$ is the value necessary to make the new physics contribution to that observable equal to the projected experimental error. Of course, a one standard deviation effect is not sufficient to either establish or exclude a given type of new physics, but it is a reasonable measure of sensitivity. Columns 6 and 7 play the same role for $\sin ^{2} \theta_{W}^{(a ; Z)}$ as columns 3 and 4 do for $O_{a}$, and in most cases the values of $\lambda^{\text {min }}$ in column 5 are also applicable to the values in columns 6 and 7 . We reemphasize that although the central values of $\sin ^{2} \theta_{W}^{a}$ extracted from each experiment depend on the renormalization scheme, both the experimental uncertainty $\Delta \sin ^{2} \theta_{W}^{\exp }$ in column 6 and the relative shift $\Delta \sin ^{2} \theta_{W}^{(a ; Z)}$ in column 7 are essentially scheme independent.

To see what experiments are sensitive to a given type of new physics $i$, for each $O_{a}$ we define the ratio of the calculated deviation from the SM due to the new physics $i$ to the projected experimental error:

$$
r_{a}^{i}(\lambda)=\frac{\left|\Delta O_{a}^{i}\right|}{\Delta O_{a}^{\text {exp }}}
$$

Although $r_{a}^{i}$ itself is dependent on the value of $\lambda$, the relative sensitivities, or the relative values of $r_{a}^{i}$ for different $O_{a}$, are independent of $\lambda$. The larger $r_{a}^{i}$ is, the more significantly the $i$-th new physics manifests itself, and the more likely for the new physics $i$ to be seen in experiments measuring $O_{a} . r_{a}^{i}(\lambda)$ is proportional to $\lambda$, with $r_{a}^{i}\left(\lambda_{a}^{\min }\right)=1$. To see the relative sensitivity of each $O_{a}$ graphically, we plot $1 / \lambda_{a}^{\min }$ from the numerical results for each type of new physics. As an example, consider Fig. 5.1, in which the new physics is an extra $Z_{x}$ with $C=\sqrt{2 / 5}$. The $x$-axis is labeled by the physical observables with their explicit definitions to be found in Section 3. The vertical bars represent the relative values of $1 / \lambda_{a}^{\min }$ for each $O_{a}$. The higher the bar, the more sensitive is $O_{a}$ to the $Z_{x}$. The values of $M_{Z_{2}}$ corresponding to $1 / \lambda^{m s n}$ are indicated on the right-hand scale. The height of each bar can be scaled up or down if the precision of the corresponding experiment varies. For example, if the error of $M_{W}$ is reduced to $50 \mathrm{MeV}$ rather than $100 \mathrm{MeV}$, then the bar of $M_{W}$ should be scaled up by a factor of two. On the other hand, if the error of $A_{L R}$ is 0.006 rather than the projected 0.004 , then the bar of $A_{L R}$ should be scaled down accordingly. At the moment, let us take the projected precision seriously. Then we see that $R_{\nu}$ is especially sensitive to this type of $Z_{x}$. Looking back to Table 5.1, at $\lambda=0.01$ the deviation in $R_{\nu}$ is some five times its projected experimental error, and $\lambda_{a}^{\min }=0.0019$, corresponding to $M_{Z_{2}} \simeq 1295 \mathrm{GeV} . M_{W}, g_{L}^{2}, \sigma_{\nu \mathrm{e}} /\left(\sigma_{\nu \mathrm{ee}}+\sigma_{\overline{\nu e}_{\mathrm{e}}}\right), C_{1+}(i s o)$, $A_{L R}(L E P), \Gamma_{i n v}, \Gamma_{l i}$, and $\Gamma_{Z}$ all have $\lambda^{m i n}<0.01$, corresponding to $M_{Z_{2}}=565 \mathrm{GeV}$.

\footnotetext{
${ }^{2}$ In some cases. such as extra $Z$ bosons, there is more than one extra parameter. However, only one is related to the coupling strength, while the others are of order unity. We pick typical values for the latter parameters for definiteness.

${ }^{3}$ That means that the anajyses based on $\sin ^{2} \theta_{W}$ and on the observables themselves are equivalent. That is true provided that the expected variation in the observable can be described by a reasonable variation in sin ${ }^{2} \theta_{W}$ and that the relation is approximately linear within the experimentally prescribed region. This condition is satisfied by all of the observables in this paper except $\theta_{L}, \theta_{R}, g_{A}^{e}$, and $C_{2 p}$, which are insensitive to $\sin ^{2} \theta_{W}$.
} 
Table 5.1: Deviations from the SM by an Extra $Z_{x}$ Boson $\left(C=(2 / 5)^{1 / 2}\right)$. Type: $Z_{\chi}$ Free parameter: $\lambda=\left(\frac{g_{2}^{3}}{M_{Z_{2}}^{2}}\right) /\left(\frac{g_{1}^{2}}{M_{Z}^{2}}\right)$ with $C=\Theta_{g_{1}} / \lambda=(2 / 5)^{1 / 2} ;$ (Section 4.1). $g_{1}, g_{2}$ and $M_{Z}, M_{Z_{2}}$ are the coupling constants and masses of the ordinary $Z$ and the extra $Z_{\chi}$ bosons, respectively; $\Theta$ is their mixing angle. Inputs: $M_{Z}=91.187 \mathrm{GeV}, m_{t}=150 \mathrm{GeV}, M_{H}=250 \mathrm{GeV},\left(\sin ^{2} \theta_{W}^{M}=0.2259, \sin ^{2} \hat{\theta}_{W}=0.2325\right)$. Comments: For the explicit definitions of physical observables, see Section 3. $\lambda^{\min }$ is the value of $\lambda$ for which the change $\Delta O_{a}$ is equal to the projected one standard deviation experimental error. The inverses of the sixth column $1 / \lambda^{\text {min }}$ are plotted in Fig. 5.1. Following the argument given in the text, the bigger $1 / \lambda_{a}^{\min }$ is, the more likely for $Z_{\chi}$ to be detected by measuring $O_{a} . R_{\nu}$ is sensitive to this particular $Z_{x}$; it has $\lambda_{a}^{\min }=0.0019$ which corresponds to $M_{Z_{3}}=1295 \mathrm{GeV}$ for $g_{2}^{2} / g_{1}^{2}=5 / 3 \sin ^{2} \theta_{W} . M_{W}, g_{L}^{2}, \sigma_{\nu e} /\left(\sigma_{\nu, e}+\sigma_{\bar{\nu} e}\right), C_{1+}(i s o), A_{L R}(L E P), \Gamma_{\text {inv }}, \Gamma_{1 I}$, and $\Gamma_{z}$ all have $\lambda^{\text {min }}<0.01$, corresponding to $M_{Z_{3}}=565 \mathrm{GeV}$.

\begin{tabular}{||c||r||r|r|r||r|r||}
\hline \hline Quantities & $O_{a}^{S M}$ & $\Delta O_{a}^{\text {exp }}$ & $\begin{array}{r}\Delta O_{a}^{i} \\
\end{array}$ & $\lambda_{a}^{\text {min }}$ & $\Delta \sin ^{2} \theta_{W}^{\text {exp }}$ & $\begin{array}{r}\Delta \sin ^{2} \theta_{W}^{(a ; Z)} \\
\lambda=0.01\end{array}$ \\
\hline \hline$M_{Z}(G e V)$ & 91.1870 & 0.0070 & - & - & 0.0003 & - \\
$M_{W}(G e V)$ & 80.2672 & 0.1050 & 0.2263 & 0.0046 & 0.0006 & -0.0013 \\
$g_{L}^{2}$ & 0.3025 & 0.0039 & 0.0074 & 0.0053 & 0.0052 & -0.0099 \\
$g_{R}^{2}$ & 0.0300 & 0.0033 & -0.0017 & 0.0198 & 0.0130 & -0.0064 \\
$R_{\nu}$ & 0.3145 & 0.0013 & 0.0067 & 0.0019 & 0.0020 & -0.0105 \\
$\theta_{L}$ & 2.4634 & 0.0350 & 0.0057 & 0.0617 & - & - \\
$\theta_{R}$ & 5.1765 & 0.4100 & -0.0323 & 0.1271 & - & - \\
$g_{V}^{e}$ & -0.0388 & 0.0170 & 0.0071 & 0.0240 & 0.0085 & 0.0035 \\
$g_{A}^{e}$ & -0.5054 & 0.0150 & -0.0001 & - & - & - \\
$\sigma_{\nu} / \sigma_{\nu}$ & 1.1636 & 0.0456 & -0.0323 & 0.0141 & 0.0050 & 0.0035 \\
$\sigma_{\nu} /\left(\sigma_{\nu}+\sigma_{\nu}\right)$ & 0.1480 & 0.0027 & -0.0037 & 0.0071 & 0.0025 & 0.0035 \\
$C_{1+}$ & 0.1285 & 0.0013 & -0.0005 & 0.0262 & 0.0033 & -0.0013 \\
$C_{1+}(i s o)$ & 0.1285 & 0.0003 & -0.0005 & 0.0068 & 0.0009 & -0.0013 \\
$C_{1-}$ & -0.3665 & 0.1000 & -0.0018 & 0.5437 & 0.0687 & -0.0013 \\
$C_{2 p}$ & -0.0145 & 0.0460 & -0.0001 & - & - & - \\
$C_{2 p}(1)$ & -0.0145 & 0.0046 & -0.0001 & 0.4374 & - & - \\
$C_{2 m}$ & -0.0588 & 0.1100 & -0.0050 & 0.2206 & 0.0273 & -0.0012 \\
$2 C_{1 u}+C_{1 d}$ & -0.0362 & 0.0040 & -0.0025 & 0.0157 & 0.0020 & -0.0013 \\
$A_{L R}(S L C)$ & 0.1399 & 0.0066 & -0.0051 & 0.0130 & 0.0008 & 0.0007 \\
$A_{L R}(L E P)$ & 0.1399 & 0.0041 & -0.0051 & 0.0081 & 0.0005 & 0.0007 \\
$A_{F B}^{p o l}(c)$ & 0.4759 & 0.0250 & 0.0048 & 0.0520 & 0.0096 & -0.0018 \\
$A_{F B}(c)$ & 0.0666 & 0.0070 & -0.0017 & 0.0401 & 0.0017 & 0.0004 \\
$A_{F B}^{p o l}(b)$ & 0.6971 & 0.0200 & 0.0044 & 0.0457 & 0.0428 & -0.0094 \\
$A_{F B}(b)$ & 0.0976 & 0.0054 & -0.0029 & 0.0184 & 0.0010 & 0.0005 \\
$A_{F B}^{p o l}(\mu)$ & 0.1050 & 0.0090 & -0.0038 & 0.0236 & 0.0015 & 0.0007 \\
$A_{F B}(\mu)$ & 0.0147 & 0.0027 & -0.0011 & 0.0253 & 0.0017 & 0.0007 \\
$A_{p o l}(\tau)$ & 0.1399 & 0.0110 & -0.0051 & 0.0216 & 0.0014 & 0.0007 \\
$\Gamma_{i n v}(G e V)$ & 0.5008 & 0.0060 & 0.0080 & 0.0075 & 0.0025 & -0.0034 \\
$\Gamma_{I I}(G e V)$ & 0.0838 & 0.0003 & -0.0004 & 0.0085 & 0.0006 & 0.0008 \\
$\Gamma_{c z}(G e V)$ & 0.2976 & 0.0300 & -0.0001 & - & 0.0155 & 0.0001 \\
$\Gamma_{b \bar{b}}(G e V)$ & 0.3763 & 0.0090 & 0.0029 & 0.0313 & 0.0040 & -0.0013 \\
$\Gamma_{Z}(G e V)$ & 2.4929 & 0.0070 & 0.0153 & 0.0046 & 0.0005 & -0.0011 \\
\hline \hline
\end{tabular}




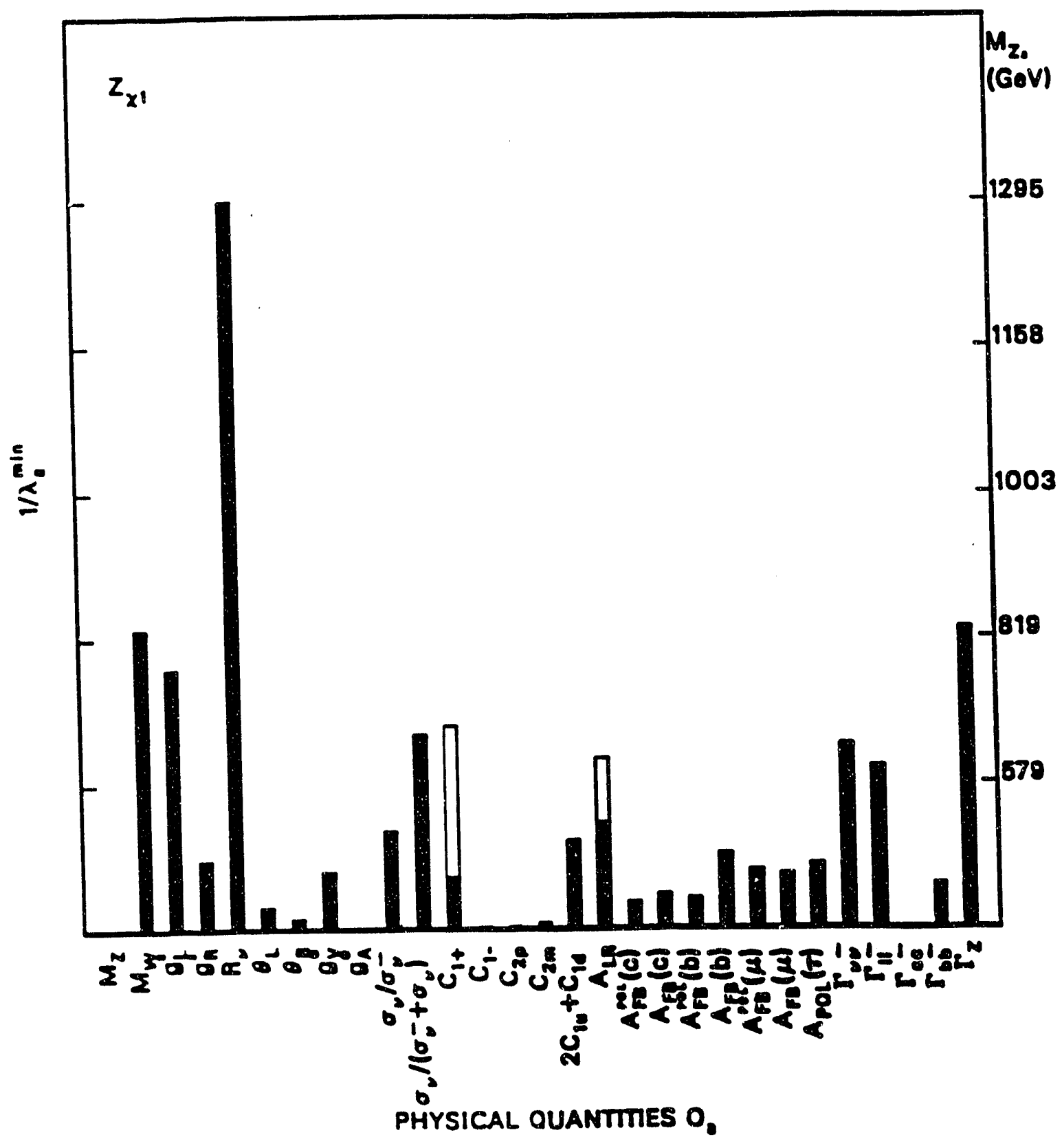

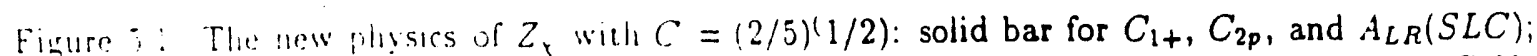

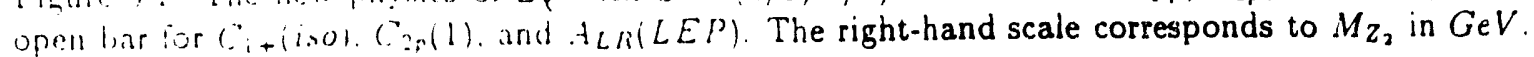


Table 5.2: Deviations from the SM by an $S U(5)$ Leptoquark $\left(\eta_{L} \neq 0\right)$. Type: $S U(5)$ Leptoquark. Free parameter: $\lambda=\frac{2^{(1 / 2)\left|\eta_{L}\right|^{2}}}{8 M_{S}^{2} G_{F}} . \quad\left(\eta_{L} \neq 0, \eta_{R}=0\right)$; (Section 4.2). Inputs: $M_{Z}=91.187 \mathrm{GeV}$, $m_{t}=150 \mathrm{GeV}, M_{H}=250 \mathrm{GeV},\left(\sin ^{2} \theta M=0.2259, \sin ^{2} \hat{\theta}_{w}=0.2325\right)$. Comments: For the explicit definitions of physical observables, see Section 3. The inverses of the sixth column $1 / \lambda^{\mathrm{min}}$ are plotted in Fig. 5.2. Leptoquarks have no effect on $Z$-pole physics, $M_{W}$ and purely leptonic processes. For $C_{1+}($ iso $), \lambda^{m i n}=0.0005$, corresponding to $\frac{M_{\mathrm{s}}}{\left|\eta_{L}\right|}>5.5 \mathrm{TeV} . C_{1+}$ and $2 C_{1 u}+C_{1 d}$ are sensitive up to $\sim 2.8 \mathrm{TeV}$. For the leptoquark with $\eta_{R} \neq 0, \eta_{L}=0$ only the atomic parity-violation coefficients are affected. The deviations on the $C$ 's are the same as those in this table but with minus signs.

\begin{tabular}{||c||r||r|r|r||c|r||}
\hline \hline Quantities & $O_{a}^{S M}$ & $\Delta O_{a}^{\text {exp }}$ & $\begin{array}{r}\Delta O_{a}^{i} \\
\lambda=0.01\end{array}$ & $\lambda_{a}^{\min }$ & $\Delta \sin ^{2} \theta_{W}^{\text {exp }}$ & $\begin{array}{r}\Delta \sin ^{2} \theta_{W}^{(a ; Z)} \\
\lambda=0.01\end{array}$ \\
\hline \hline$M_{Z}(\mathrm{GeV})$ & 91.1870 & 0.0070 & - & - & 0.0003 & - \\
$g_{L}^{2}$ & 0.3025 & 0.0039 & 0.0025 & 0.0155 & 0.0052 & -0.0034 \\
$g_{R}^{2}$ & 0.0300 & 0.0033 & -0.0006 & 0.0550 & 0.0130 & -0.0023 \\
$R_{\nu}$ & 0.3145 & 0.0013 & 0.0023 & 0.0056 & 0.0020 & -0.0035 \\
$\theta_{L}$ & 2.4634 & 0.0350 & 0.0114 & 0.0307 & - & - \\
$C_{1+}$ & 0.1285 & 0.0013 & -0.0067 & 0.0020 & 0.0033 & -0.0169 \\
$C_{1+}(i s o)$ & 0.1285 & 0.0003 & -0.0067 & 0.0005 & 0.0009 & -0.0169 \\
$C_{1-}$ & -0.3665 & 0.1000 & -0.0075 & 0.1339 & 0.0687 & -0.0051 \\
$C_{2 p}$ & -0.0145 & 0.0460 & -0.0100 & 0.0460 & - & - \\
$C_{2 p}(1)$ & -0.0145 & 0.0046 & -0.0100 & 0.0046 & - & - \\
$C_{2 m}$ & -0.0588 & 0.1100 & -0.0100 & 0.1100 & 0.0273 & -0.0025 \\
$2 C_{1 u}+C_{1 d}$ & -0.0362 & 0.0040 & -0.0200 & 0.0020 & 0.0020 & -0.0099 \\
\hline \hline
\end{tabular}




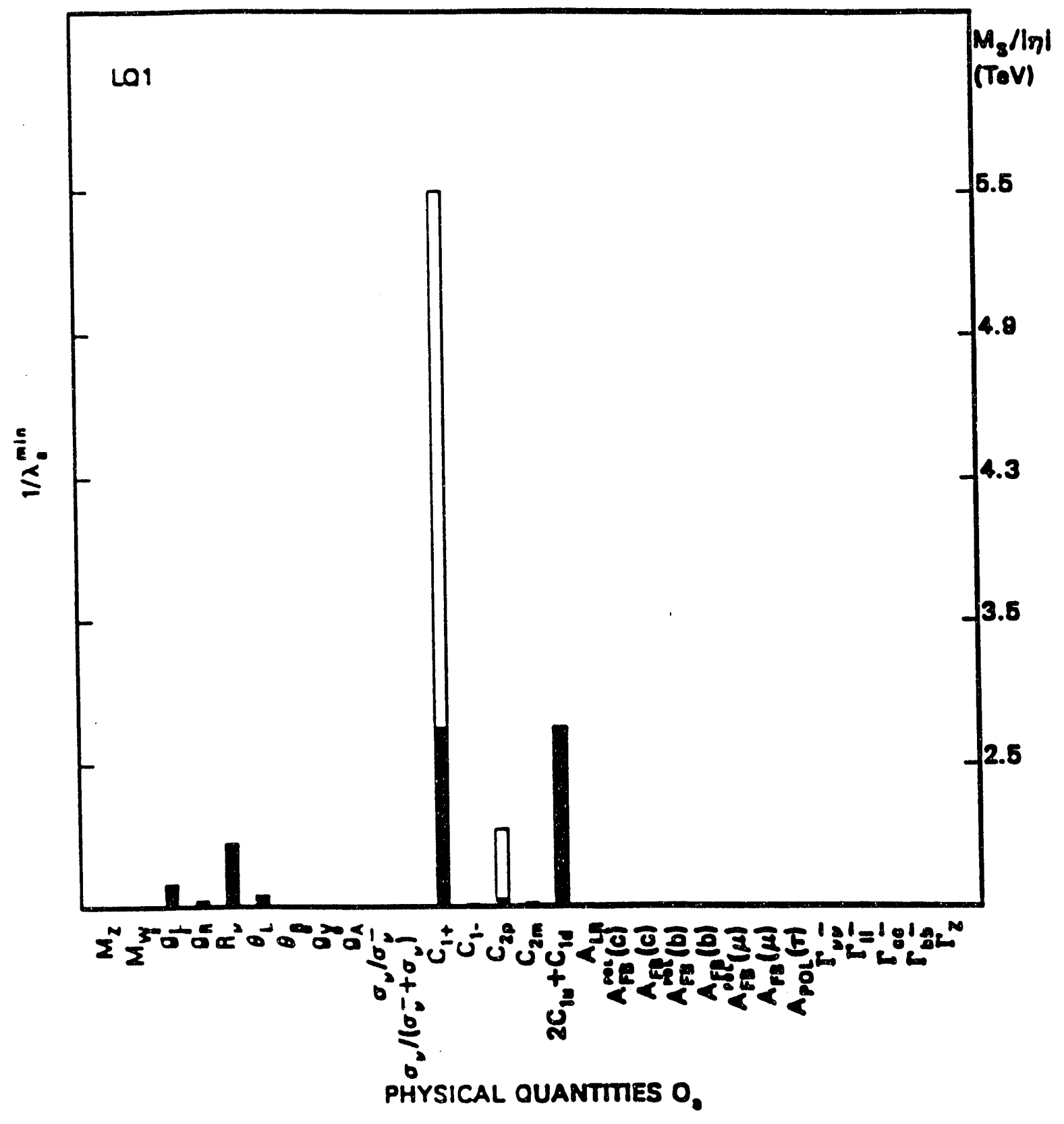

Firure : :- The new physics of $S\left[(5)\right.$ leptoruark. type 1: solid bar for $C_{1+}$ and $C_{2 p}$; open bar for $(\because+i n) 1$ anci $(\because, 1)$ The rigin-hind scale corresponds to $M_{S} /|\eta|$ in $T e V$. 
Table 5.3: Deviations from the SM by an Extra Fermion $\left(u_{L}\right)$. Type: extra left-handed $Q=2 / 3$, $S U(2)$-singlet quark; (Section 4.3). Free parameter: $\lambda=\sin ^{2} \theta_{L}^{u}$, where $\theta_{L}^{u}$ is the mixing angle between ordinary and exotic $\left(S U(2)\right.$-singlet) left-handed $u$-quark. Inputs: $M_{Z}=91.187 \mathrm{GeV}$, $m_{t}=150 \mathrm{GeV}, M_{H}=250 \mathrm{GeV},\left(\sin ^{2} \theta_{W}^{M}=0.2259, \sin ^{2} \hat{\theta}_{W}=0.2325\right)$. Comments: For the explicit definitions of physical observables, see Section 3. The deviations of $A_{F B}^{p o l}(c), A_{F B}(c)$ and $\Gamma_{c \bar{c}}$ are due to the the mixing between the ordinary $c$-quark and an exotic $c$-quark. For these the mixing angle is $\sin ^{2} \theta_{L}^{c}$ rather than $\sin ^{2} \theta_{L}^{u}$. The inverses of the sixth column $1 / \lambda^{\min }$ are plotted in Fig. 5.3. Following the argument given in the text, the bigger $1 / \lambda_{a}^{\min }$ is, the more likely for the extra $u_{L}$ to be detected by measuring $O_{a} . C_{1+}($ iso $)$ is sensitive to $\sin ^{2} \theta_{L}^{u} \sim 0.0010$, while $R_{\nu}, C_{1+}$, and $2 C_{1 u}+C_{1 d}$ have $\lambda^{\text {min }} \sim 0.004$.

\begin{tabular}{||c||r||r|r|r||c|r||}
\hline \hline Quantities & $O_{a}^{S M}$ & $\Delta O_{a}^{\text {exp }}$ & $\begin{array}{r}\Delta O_{a}^{i} \\
\lambda=0.01\end{array}$ & $\lambda_{a}^{\min }$ & $\Delta \sin ^{2} \theta_{W}^{\text {exp }}$ & $\begin{array}{r}\Delta \sin ^{2} \theta_{W}^{(a ; Z)} \\
\lambda=0.01\end{array}$ \\
\hline \hline$M_{Z}(G e V)$ & 91.1870 & 0.0070 & - & - & 0.0003 & - \\
$g_{L}^{2}$ & 0.3025 & 0.0039 & -0.0035 & 0.0113 & 0.0052 & 0.0046 \\
$R_{\nu}$ & 0.3145 & 0.0013 & -0.0035 & 0.0037 & 0.0020 & 0.0054 \\
$\theta_{L}$ & 2.4634 & 0.0350 & 0.0071 & 0.0494 & - & - \\
$C_{1+}$ & 0.1285 & 0.0013 & 0.0034 & 0.0039 & 0.0033 & 0.0085 \\
$C_{1+}(i s o)$ & 0.1285 & 0.0003 & 0.0034 & 0.0010 & 0.0009 & 0.0085 \\
$C_{1-}$ & -0.3665 & 0.1000 & 0.0038 & 0.2649 & 0.0687 & 0.0026 \\
$C_{2 p}$ & -0.0145 & 0.0460 & 0.0004 & - & - & - \\
$C_{2 p}(1)$ & -0.0145 & 0.0046 & 0.0004 & 0.1186 & - & - \\
$C_{2 m}$ & -0.0588 & 0.1100 & 0.0004 & - & 0.0273 & 0.0001 \\
$2 C_{1 u}+C_{1 d}$ & -0.0362 & 0.0040 & 0.0101 & 0.0040 & 0.0020 & 0.0050 \\
$A_{F B}^{\text {pol }}(c)$ & 0.4759 & 0.0250 & -0.0061 & 0.0412 & 0.0096 & 0.0023 \\
$A_{F B}(c)$ & 0.0666 & 0.0070 & -0.0008 & 0.0825 & 0.0017 & 0.0002 \\
$\Gamma_{c \bar{c}}(\mathrm{GeV})$ & 0.2976 & 0.0300 & -0.0069 & 0.0437 & 0.0155 & 0.0035 \\
$\Gamma_{Z}(\mathrm{GeV})$ & 2.4929 & 0.0070 & -0.0069 & 0.0102 & 0.0005 & 0.0005 \\
\hline \hline
\end{tabular}




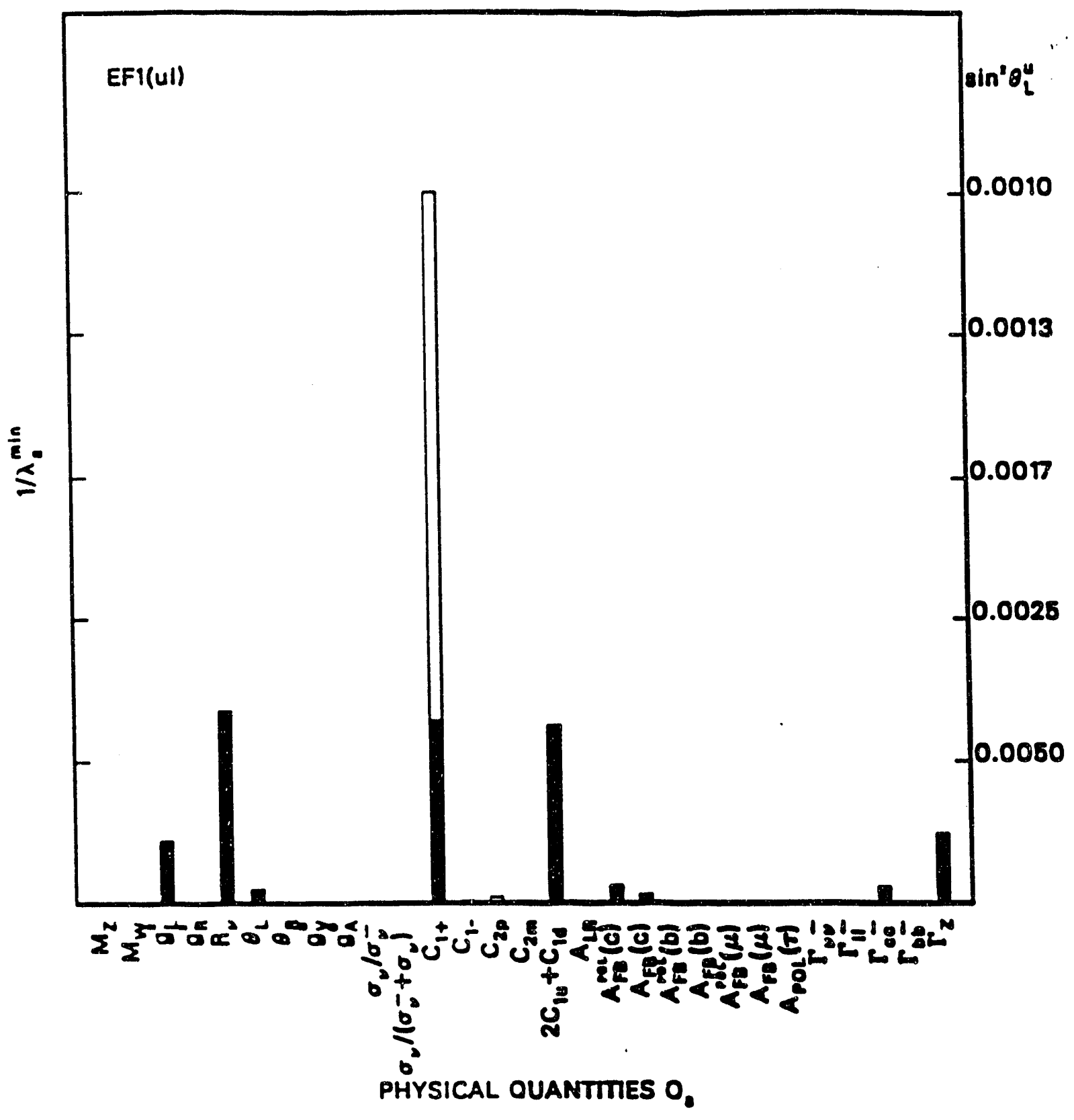

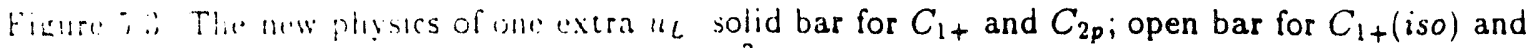
( $\because$ 1) The ilsill-hand enale corresponds $10 \sin ^{2} \theta_{L}^{u}$. 
Table 5.4: Deviations from the SM due to Heavy Particle Contributions to Loop Corrections $\left(h_{V}\right)$. Type: Deviations arising from loop corrections due to heavy particle physics, e.g., non-degenerate heavy fermion or boson multiplets, with $h_{V} \neq 0, h_{A Z}=0, h_{A W}=0$; (Section 4.5). Free parameter: $\lambda=\alpha h_{V}(\alpha=1 / 137.036)$ Inputs: $M_{Z}=91.187 \mathrm{GeV}, m_{t}=150 \mathrm{GeV}, M_{H}=250 \mathrm{GeV},\left(\sin ^{2} \theta_{W}^{M}=\right.$ $\left.0.2259, \sin ^{2} \hat{\theta}_{W}=0.2325\right)$. Comments: For the explicit definitions of physical observables, see Section 3. The inverses of the sixth column $1 / \lambda^{\mathrm{min}}$ are plotted in Fig. 5.4. The deviation pattern among the observables is exactly the same as that of non-standard Higgs fields. $R_{\nu}$ has $\lambda^{\text {min }}=$ 0.0015 , corresponding to $h_{V}=0.21 ; A_{L R}(L E P)$ has $\lambda^{\mathrm{min}}=0.0017\left(h_{V}=0.23\right)$; and $M_{W}$ has $\lambda^{\text {min }}=0.0019\left(h_{V}=0.26\right) . A_{L R}(S L C), A_{F B}(b), \sigma_{\nu} /\left(\sigma_{\bar{\nu}}+\sigma_{\nu}\right), \Gamma_{l i}$, and $\Gamma_{Z}$ all have $\lambda^{\text {min }}<0.004$.

\begin{tabular}{|c|c|c|c|c|c|c|}
\hline Quantities & $O_{a}^{S M}$ & $\Delta O_{a}^{\exp }$ & $\begin{array}{r}\Delta O_{a}^{i} \\
\lambda=0.01 \\
\end{array}$ & $\lambda_{a}^{\min }$ & $\Delta \sin ^{2} \theta_{W}^{\exp }$ & $\begin{array}{r}\Delta \sin ^{2} \theta_{W}^{(a ; 2)} \\
\lambda=0.01\end{array}$ \\
\hline$\overline{M_{Z}(G e V)}$ & 91.1870 & 0.0070 & $\overline{-}$ & - & 0.0003 & - \\
\hline$M_{W}(G e V)$ & 80.2672 & 0.1050 & 0.5657 & $0.6 u 19$ & 0.0006 & -0.0032 \\
\hline$g_{L}^{2}$ & 0.3025 & 0.0039 & 0.0084 & 0.0046 & 0.0052 & -0.0113 \\
\hline$g_{R}^{2}$ & 0.0300 & 0.0033 & -0.0002 & 0.1503 & 0.0130 & -0.0009 \\
\hline$R_{v}$ & 0.3145 & 0.0013 & 0.0083 & 0.0015 & 0.0020 & -0.0130 \\
\hline$\theta_{L}$ & 2.4634 & 0.0350 & -0.0018 & 0.1956 & - & - \\
\hline$\theta_{R}$ & 5.1765 & 0.4100 & 0.0000 & - & - & 1000.0000 \\
\hline$g_{V}^{e}$ & -0.0388 & 0.0170 & -0.0067 & 0.0252 & 0.0085 & -0.0034 \\
\hline$g_{A}^{e}$ & -0.5054 & 0.0150 & -0.0051 & 0.0297 & - & - \\
\hline$\sigma_{\nu} / \sigma_{D}$ & 1.1636 & 0.0456 & 0.0289 & 0.0158 & 0.0050 & -0.0032 \\
\hline$\sigma_{\nu} /\left(\sigma_{\bar{\nu}}+\sigma_{\nu}\right)$ & 0.1480 & 0.0027 & 0.0068 & 0.0039 & 0.0025 & -0.0064 \\
\hline$C_{1+}$ & 0.1285 & 0.0013 & 0.0000 & 0.3817 & 0.0033 & 0.0001 \\
\hline$C_{1+(\text { iso })}$ & 0.1285 & 0.0003 & 0.0000 & 0.0992 & 0.0009 & 0.0001 \\
\hline$C_{1-}$ & -0.3665 & 0.1000 & -0.0083 & 0.1207 & 0.0687 & -0.0057 \\
\hline$C_{2 p}$ & -0.0145 & 0.0460 & -0.0001 & - & - & - \\
\hline$C_{2 p}(1)$ & -0.0145 & 0.0046 & -0.0001 & 0.3180 & - & - \\
\hline$C_{2 m}$ & -0.0588 & 0.1100 & -0.0134 & 0.0824 & 0.0273 & -0.0033 \\
\hline $2 C_{l u}+C_{l d}$ & -0.0362 & 0.0040 & -0.0068 & 0.0059 & 0.0020 & -0.0034 \\
\hline$A_{L R}(S L C)$ & 0.1399 & 0.0066 & 0.0247 & 0.0027 & 0.0008 & -0.0032 \\
\hline$A_{L R}(L E P)$ & 0.1399 & 0.0041 & 0.0247 & 0.0017 & 0.0005 & -0.0032 \\
\hline$A_{F B}^{p o l}(c)$ & 0.4759 & 0.0250 & 0.0083 & 0.0302 & 0.0096 & -0.0032 \\
\hline$A_{F B}(c)$ & 0.0666 & 0.0070 & 0.0129 & 0.0054 & 0.0017 & -0.0032 \\
\hline$A_{F B}^{p o l}(b)$ & 0.6971 & 0.0200 & 0.0015 & 0.1347 & 0.0428 & -0.0032 \\
\hline$A_{F B}(b)$ & 0.0976 & 0.0054 & 0.0174 & 0.0031 & 0.0010 & -0.0032 \\
\hline$A_{F B}^{p o l}(\mu)$ & 0.1050 & 0.0090 & 0.0185 & 0.0049 & 0.0015 & -0.0032 \\
\hline$A_{F B}(\mu)$ & 0.0147 & 0.0027 & 0.0052 & 0.0052 & 0.0017 & -0.0032 \\
\hline$A_{p o l}(\tau)$ & 0.1399 & 0.0110 & 0.0247 & 0.0045 & 0.0014 & -0.0032 \\
\hline$\Gamma_{i n v}(\mathrm{GeV})$ & 0.5008 & 0.0060 & 0.0050 & 0.0120 & 0.0025 & -0.0021 \\
\hline$\Gamma_{1 i}(\mathrm{GeV})$ & 0.0838 & 0.0003 & 0.0010 & 0.0029 & 0.0006 & -0.0023 \\
\hline$\Gamma_{c \bar{c}}(\mathrm{GeV})$ & 0.2976 & 0.0300 & 0.0047 & 0.0644 & 0.0155 & -0.0024 \\
\hline$\Gamma_{b \dot{b}}(\mathrm{GeV})$ & 0.3763 & 0.0090 & 0.0052 & 0.0172 & 0.0040 & -0.0023 \\
\hline$\Gamma_{Z}(\mathrm{GeV})$ & 2.4929 & 0.0070 & 0.0333 & 0.0021 & 0.0005 & -0.0023 \\
\hline
\end{tabular}




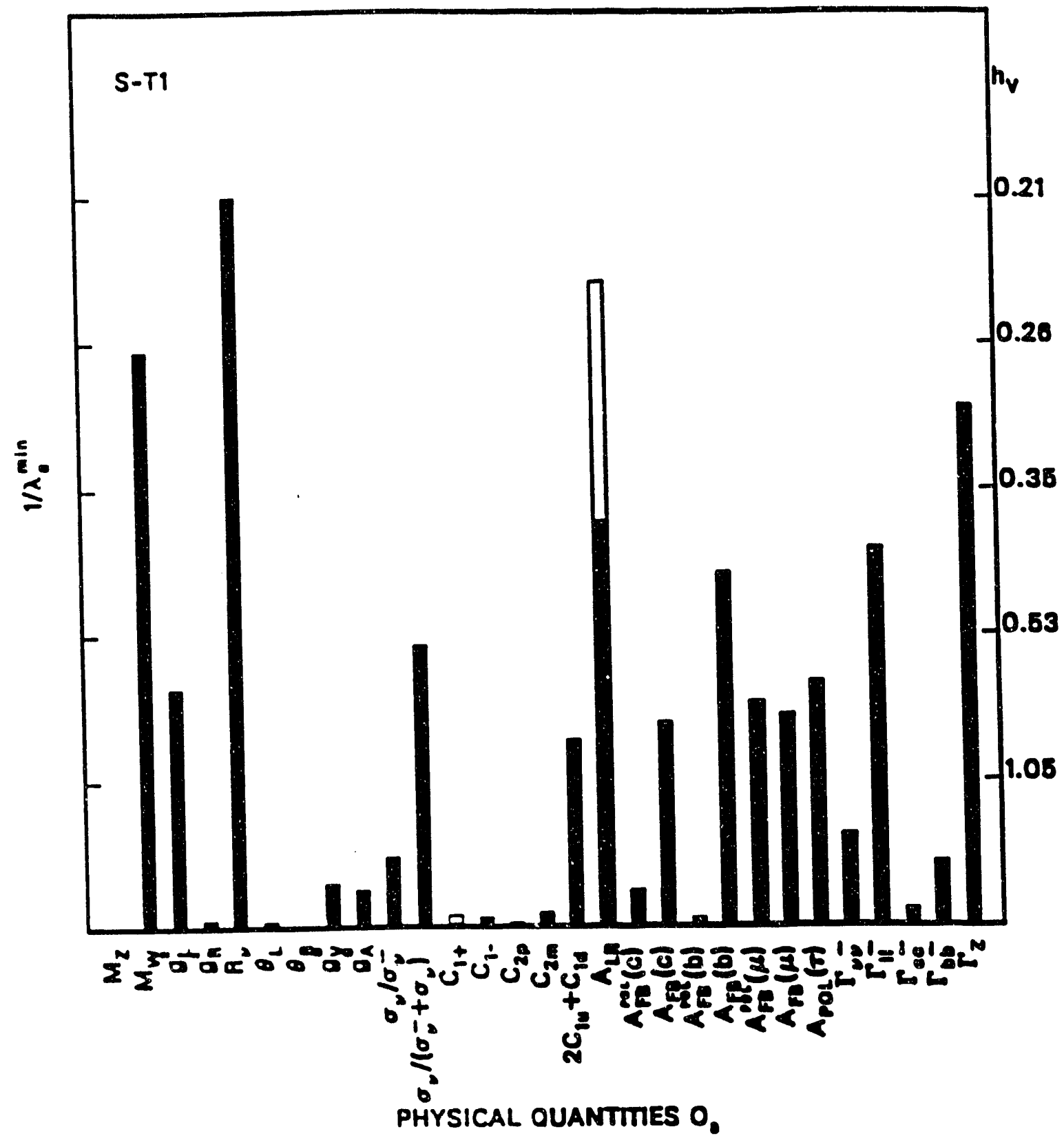

Figure it ll.3ry physics through loop corrections $\left(h_{v}\right)$ : solid bar for $C_{1+}, C_{2 p}$, and $A_{L R}(S L C)$; upen har for $\ldots+1(10) 1$. 
Table 5.5: Deviations from the SM due to Four-Fermi Operators induced by Compositeness. Type: four-fermi operators induced by compositeness of fermions; (Section 4.4). Free parameter: $\lambda=$ $\frac{2(1 / 2) x}{G \Lambda^{2}}$ Inputs: $M_{Z}=91.187 \mathrm{GeV}, m_{t}=150 \mathrm{GeV}, M_{H}=250 \mathrm{GeV},\left(\sin ^{2} \theta_{W}^{M}=0.2259, \sin ^{2} \hat{\theta}_{W}=\right.$ $0.2325)$. Comments: For the explicit definitions of physical observables, see Section 3. In this table are included three types of operators: (1) $-L_{1}= \pm \frac{4 \pi}{\Lambda_{1}^{j}} \bar{l}_{\mu L} \gamma^{\mu} l_{\mu L} \bar{q}_{L} \gamma_{\mu} q_{L}$, which shifts $\epsilon_{L}(u), \epsilon_{L}(d), C_{2 u}$, and $C_{2 d} ;(2)-L_{2}= \pm \frac{4 \pi}{\Lambda_{2}^{3}} \bar{\nu}_{\mu L} \gamma^{\mu} \nu_{\mu L} \bar{e}_{L} \gamma_{\mu} e_{L}$, which shifts $g_{V}^{e}$ and $g_{A}^{e} ;(j)-L_{3}= \pm \frac{4 \pi}{\Lambda_{3}^{e}} \bar{e}_{L} \gamma^{\mu} e_{L} \bar{q}_{L} \gamma_{\mu} q_{L}$, which shifts $C_{1 u}$ and $C_{1 d}$. The deviation pattern of $L_{1}$ is plotted in Figure 5.5. $C_{2 p}(1)$ would be sensitive to $\lambda^{\mathrm{min}}=0.0023$, corresponding to $\Lambda_{1}=12.9 \mathrm{TeV}$.

\begin{tabular}{||c||r||r|r|r||c|r||}
\hline \hline Quantities & $O_{a}^{S M}$ & $\Delta O_{a}^{\text {exp }}$ & $\begin{array}{r}\Delta O_{a}^{i} \\
\lambda=0.01\end{array}$ & $\lambda_{a}^{\min }$ & $\Delta \sin ^{2} \theta_{W}^{\text {exp }}$ & $\begin{array}{r}\Delta \sin ^{2} \theta_{W}^{(a ; Z)} \\
\lambda=0.01\end{array}$ \\
\hline \hline$M_{Z}(G e V)$ & 91.1870 & 0.0070 & - & - & 0.0003 & - \\
$g_{L}^{2}$ & 0.3025 & 0.0039 & -0.0017 & 0.0234 & 0.0052 & 0.0022 \\
$R_{\nu}$ & 0.3145 & 0.0013 & -0.0017 & 0.0077 & 0.0020 & 0.0026 \\
$\theta_{L}$ & 2.4634 & 0.0350 & -0.0256 & 0.0137 & - & - \\
$g_{V}^{e}$ & -0.0388 & 0.0170 & 0.0100 & 0.0170 & 0.0085 & 0.0050 \\
$g_{A}^{e}$ & -0.5054 & 0.0150 & 0.0100 & 0.0150 & - & - \\
$\sigma_{\nu} / \sigma_{\bar{\nu}}$ & 1.1636 & 0.0456 & -0.0421 & 0.0108 & 0.0050 & 0.0046 \\
$\sigma_{\nu} /\left(\sigma_{\bar{\nu}}+\sigma_{\nu}\right)$ & 0.1480 & 0.0027 & -0.0083 & 0.0032 & 0.0025 & 0.0078 \\
$C_{1+}$ & 0.1285 & 0.0013 & 0.0141 & 0.0009 & 0.0033 & 0.0358 \\
$C_{1+}+(i s o)$ & 0.1285 & 0.0003 & 0.0141 & 0.0002 & 0.0009 & 0.0358 \\
$C_{1-}$ & -0.3665 & 0.1000 & 0.0008 & - & 0.0687 & 0.0006 \\
$C_{2 p}$ & -0.0145 & 0.0460 & 0.0200 & 0.0230 & - & - \\
$C_{2 p}(1)$ & -0.0145 & 0.0046 & 0.0200 & 0.0023 & - & - \\
$2 C_{1 u}+C_{1 d}$ & -0.0362 & 0.0040 & 0.0300 & 0.0013 & 0.0020 & 0.0148 \\
\hline \hline
\end{tabular}




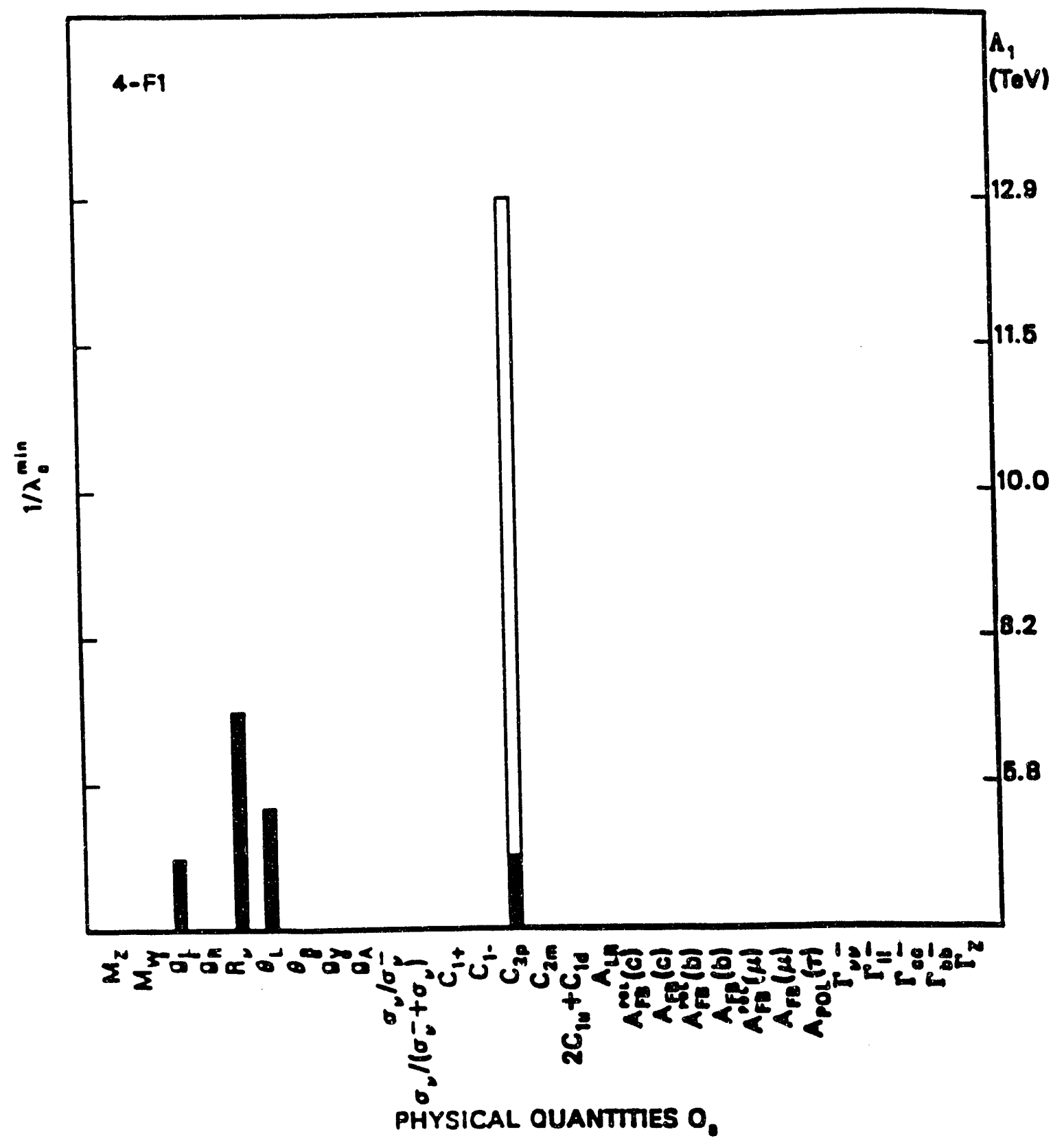

Firure ; ; The new physics of four-fermu operator, type I: solid bar for $C_{2 p}$, open bar for $C_{2 p}(1)$. Thic richlinani scale corresponds to the compositeness scale $\Lambda T e V$. 
Other figures are organized in the same form, and conclusions related to each are presented in the Comments at the bottom of the corresponding table.

In Table E.2 and Figure 5.2, the new physics is an $S U(5)$ leptoquark with $\eta_{L} \neq 0$ but $\eta_{R}=0$. Leptoquarks have no effect on $Z$-pole physics, $M_{W}$ and purely leptonic processes. For $C_{1+}($ iso $), \lambda^{\mathrm{min}}=0.0005$, corresponding to $\frac{M_{s}}{\left|\eta_{L}\right|}>5.5 \mathrm{TeV} . C_{1+}$ and $2 C_{14}+C_{16}$ are sensitive up to $\sim 2.8 \mathrm{TeV}$. For the leptoquark with $\eta_{R} \neq 0, \eta_{L}=0$ only the itomic parity-violation coefficients are affected. The deviations on the $C$ 's are the same as those in this table but with minus signs.

In Table 5.3 and Figure 5.3, the new physics is an exotic ( $S U(2)$-singlet) lefthanded $u$ quark. $C_{1+}($ iso $)$ is sensitive to $\sin ^{2} \theta_{L}^{u} \sim 0.0010$, while $R_{\nu}, C_{1+}$, and $2 C_{1 u}+C_{1 d}$ have $\lambda^{\min } \sim 0.004$.

In Table 5.4 and Figure 5.4, the deviations arising from loop corrertions due to heavy particle physics, e.g., non-degenerate heavy fermion or boson multiplets, with $h_{V} \neq 0, h_{A Z}=0, h_{A W}=0$. The deviation pattern among the rbservables is exactly the same as that of non-standard Higgs fields. $R_{\nu}$ has $\lambda^{m s n}=0.0015$, corresponding to $h_{V}=0.21 ; A_{L R}(i E P)$ has $\lambda^{\min }=0.0017\left(h_{V}=0.23\right)$; and $M_{W}$ has $\lambda^{\text {vin }}=0.0018\left(h_{V}=0.25\right) . A_{L R}(S L C), A_{F B}(b), \sigma_{\nu} /\left(\sigma_{\bar{\nu}}+\sigma_{\nu}\right), \Gamma_{1 \bar{l}}$, and $\Gamma_{Z}$ all have $\lambda^{\text {min }}<$ 0.004 .

In Table 5.5 and Figure 5.5, the new physics cre four-fermi operators induced by compositeness. In this table are included three types of operators: (1) $-L_{1}=$ $\pm \frac{4 \pi}{\Lambda_{1}^{2}} \bar{l}_{\mu L} \gamma^{\mu} l_{\mu L} \bar{q}_{L} \gamma_{\mu} q_{\Sigma}$, which shifts $\epsilon_{L}(u), \epsilon_{L}(d), C_{2 u}$, and $C_{\tilde{z} \dot{a}} ;(2)-L_{2}= \pm \frac{4 \pi}{\Lambda_{2}^{2}} \bar{\nu}_{\mu L} \gamma^{\mu} \nu_{\mu L} \bar{e}_{L} \gamma_{\mu} e_{L}$, which shifts $g_{V}^{e}$ nnd $g_{A}^{e} ;(3)-L_{3}= \pm \frac{4 \pi}{\Lambda_{3}^{2}} \bar{e}_{L} \gamma^{\mu} e_{L} \bar{q}_{L} \gamma_{\mu} q_{L}$, which shifts $C_{1 u}$ and $C_{1 d}$. The deviation patterr. of $L_{1}$ is plotted in Figure 5.5. $C_{2 p}(1)$ would be sensitive to $\lambda^{\text {min }}=$ 0.0023 , corresponding to $\Lambda_{1}=12.9 \mathrm{TeV}$.

In similar manner, we can update analysis for all of the 27 specific new physics consider $d$ in [1]. A brief surnmary of the results is given in Tables 5.6-5.9. For each types oi new physics considered are given the smallest value of $\lambda^{\text {min }}$ for any of the ohservables, the corresponding scale of new physics (e.g., $M_{Z_{3}}$ for an extra $Z$ ), and the most sensitive observables. Many of the observables are sensitive to several types of new physics with characteristic scale extending into the $\mathrm{TeV}$ range. No one observabie is the most sensitive to all types.

The specific predictions of sensitivity depend on the projected experimental uncertainties, and it is likely that many of the experiments will be more or less precise than anticipated. While keeping this caveat in mind, it is apparent from the Tables 5.1-5.9 that the observables $M_{W}, R_{\nu}, C_{1+}($ iso $), A_{L R}(L E P)$, and $\Gamma_{Z}$ are especially promising, each showing considerable sensitivity to a variety of new physics. The observables $\sigma_{\nu} /\left(\sigma_{\nu}+\sigma_{\nu_{e}}\right), A_{F B}(b), A_{p o l}(\tau), \Gamma_{l l}$, and the existing $g_{L}^{2}$ are also sensitive; most of the remaining observables are strong probes of one or more types of new physics. New physics at the $\mathrm{TeV}$ scale or higher may be observed or excluded if a significant number of these observables are measured precisely. If all the measured values are consistent with t'ie SM predictions then, except for the unlikely possibility of fine-tuned cancellations, various types of new physics will be excluded at scales of the order of those shown in Tables 5.6-5.9. 
Table 5.6: Summary of the discussion in section 5: implications for extra $Z$ bosons. The smallest $\lambda^{m i n}$ is the value of the overall coupling constant $\lambda^{\min }=\frac{g_{2}^{2}}{M_{Z_{2}}^{2}} / \frac{g_{1}^{2}}{M_{Z}^{2}}$ at which the extra $Z$ is manifested in the most sensitive observable. Here $g_{1}, g_{2}, M_{Z}$, and $M_{z_{2}}$ are the coupling constants and masses of the ordinary $Z$ and extra $Z_{2}$, and $\frac{q_{2}^{2}}{g_{1}^{2}}=\frac{5}{3} \sin ^{2} \theta_{W}$ is assumed.

\begin{tabular}{|c|c|c|c|}
\hline New Physics & Smallest $\lambda^{\min }$ & $\begin{array}{c}\text { Implications } \\
\mathrm{M}_{Z_{2}}(\mathrm{GeV})\end{array}$ & Most Sensitive Observables \\
\hline$Z_{x}(C=\sqrt{2 / 5)}$ & 0.0019 & $\sim 1295$ & $\begin{array}{l}R_{\nu} ; \\
\sigma_{\nu e} /\left(\sigma_{\nu_{e} e}+\sigma_{\nu e}\right), M_{W}, g_{L}^{2}, \Gamma_{Z}, \\
C_{1+}(i s o), A_{L E P}^{(L E P)}, \Gamma_{i n v}, \Gamma_{I J}\end{array}$ \\
\hline$Z_{x}(C=0)$ & 0.0011 & $\sim 1700$ & $\begin{array}{l}C_{1+}(i s o) \\
C_{1+}, \sigma_{\nu} /\left(\sigma_{\nu}+\sigma_{\nu}\right), 2 C_{1 u}+C_{1 d}\end{array}$ \\
\hline$Z_{\psi}(C=\sqrt{2 / 3})$ & 0.0017 & $\sim 1370$ & $\begin{array}{l}R_{\nu} ; \\
M_{W}, C_{1+}(i s o), A_{L R}, A_{F B}(b), \Gamma_{Z}\end{array}$ \\
\hline$Z_{\psi}(C=-\sqrt{2 / 3})$ & 0.0017 & $\sim 1370$ & $\begin{array}{l}\Gamma_{1 i} ; \\
M_{W}, R_{v}, C_{1+}(i s o), A_{L R}, A_{F B}(b)\end{array}$ \\
\hline$Z_{\psi}(C=0)$ & 0.025 & $\sim 357$ & $\sigma_{\nu} /\left(\sigma_{D}+\sigma_{\nu}\right)$ \\
\hline$Z_{\eta}\left(C=-\frac{1}{\sqrt{15}}\right)$ & 0.0075 & $\sim 650$ & $A_{L R}^{(L E P)}$ \\
\hline$Z_{\eta}\left(C=\frac{4}{\sqrt{15}}\right)$ & 0.0008 & $\sim 2000$ & $\begin{array}{l}C_{2 p}(1), A_{L R}^{(S L C)}, A_{F B}(b) \\
C_{1+}(i s o) ; M_{W}, R_{\nu}, \Gamma_{l I}, \Gamma_{Z}\end{array}$ \\
\hline$Z_{\eta}(C=0)$ & 0.0045 & $\sim 840$ & $C_{1+}($ iso $) ; C_{2 p}(1)$ \\
\hline$Z_{3 R}(C=\sqrt{3 / 5} \alpha)$ & 0.0009 & $\sim 1880$ & $\begin{array}{l}R_{\nu}, A_{L R}^{(L E P)} ; \\
M_{W}, A_{L R}^{(S L C)}, C_{1+}(\text { iso }), A_{F B}(b), \Gamma_{Z}\end{array}$ \\
\hline $\begin{array}{c}Z_{3 R}(C=-\sqrt{3 / 5} / \alpha) \\
Z_{3 R}(C=0)\end{array}$ & $\begin{array}{l}0.0008 \\
0.0010\end{array}$ & $\begin{array}{r}\sim 2000 \\
\sim 1785 \\
\end{array}$ & $\begin{array}{l}C_{1+}(\text { iso }) ; C_{1+}, \Gamma_{11} \\
C_{1+}(\text { iso }) ; C_{1+} \\
\end{array}$ \\
\hline
\end{tabular}

If, however, deviations are observed, then as much information as possible will be needed to confirm the discrepancy and to determine the nature of the new physics and its strength. From the pattern of the deviations of the various observables it will be possible in most cases to distinguish which type of new physics is responsible, or at least which is most likely out of the set of possibilities considered here. The deviation pattern can also distinguish true new physics from values of $m_{t}$ and $M_{H}$ different from the reference values used in the SM predictions. Identification of the origin of deviations is another reason for carrying out a large variety of precision experiments.

To quantify this, it is useful to consider an $n$-dimensional vector space, each point of which represents the possible outcome of $n$ precise experiments. Let us choose the origin in this space as the SM prediction for the $n$ observables. Then one can define an $n$-dimensional vector with components:

$$
V_{a}^{\text {exp }}=\frac{O_{a}^{\text {exp }}-O_{a}^{S M}}{\Delta O_{a}^{\text {exp }}}
$$

where $V_{a}^{\text {exp }}$ is the deviation of the $a^{\text {th }}$ observable from the the SM prediction, 
Table 5.7: Summary of the discussion in section 5: implications for exotic fermions. The smallest value $\lambda^{\min }$ is the value of $\left(\sin ^{2} \theta_{L, R}^{i}\right)^{2}$ at which the exotic fermion is manifested in the most sensitive observable. Here $\theta_{L, R}^{i}$ is the mixing angle between the ordinary and exotic fermions.

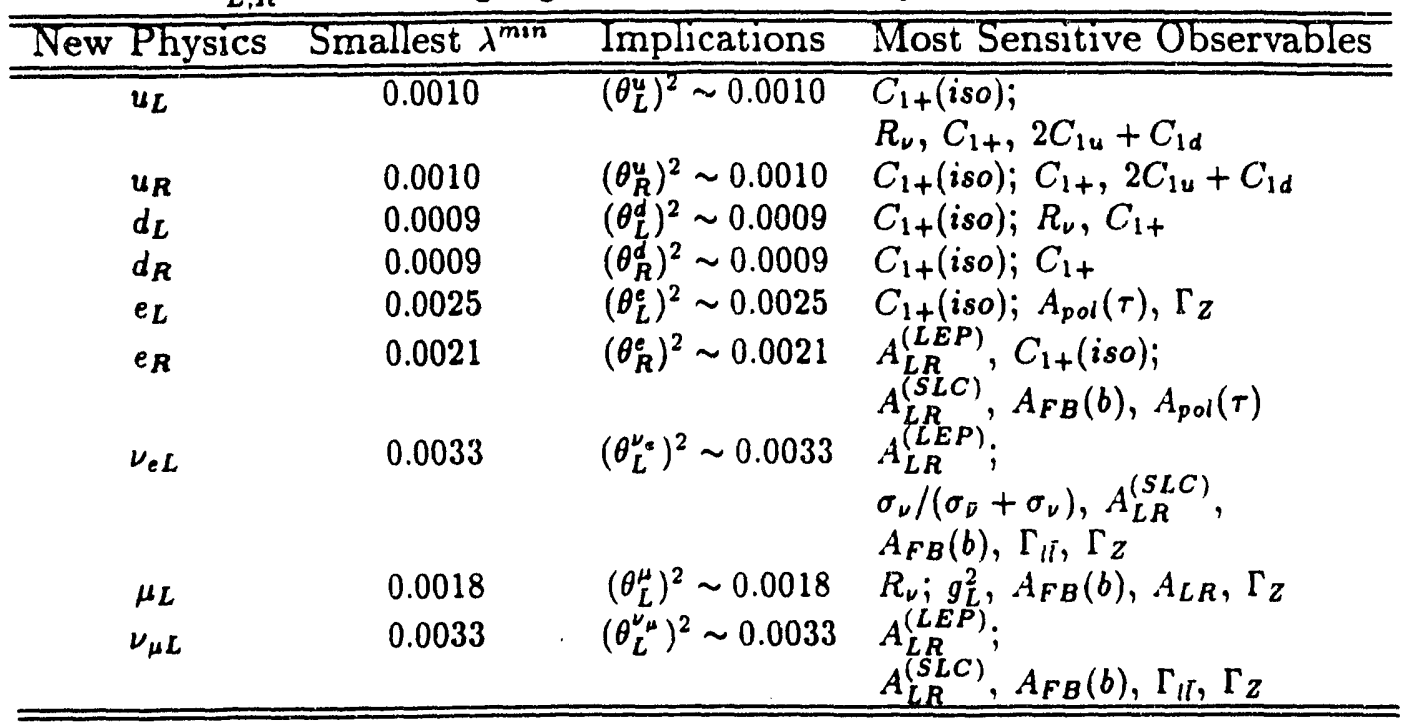

weighted by the inverse of the experimental uncertainty. Similarly, each type $i$ of new physics predicts a deviation vector with components:

$$
V_{a}^{i}(\lambda)=\frac{\Delta O_{a}^{i}(\lambda)}{\Delta O_{a}^{\operatorname{cxp}}}, a=1, \ldots, n
$$

which has a magnitude proportional to the coupling strength $\lambda$ and a direction depending on $a$. If $V_{a}^{\text {exp }}$ is compatible with zero (i.e., the total length is of order $n$ and no single component is much bigger than unity), there is no evidence for new physics. One can then set upper limits on the magnitude of each $V_{a}^{i}(\lambda)$ and therefore on each $\lambda$. On the other hand, if $V_{a}^{\exp } \gg n$ then there is evidence for new physics. The most likely type or types can be determined, at least among the possibilities considered, by choosing $i$ such that $V_{a}^{i}(\lambda)$ and $V_{a}^{\text {exp }}$ are approximately parallel. The strength $\lambda$ could be estimated from the requirement that $V_{a}^{i}(\lambda)=V_{a}^{\exp }$.

The observables considered in this paper would usually be sufficient to distinguish the nature of new physics from the directions of $V_{a}^{i}(\lambda)$. Rather than grapple further with an $n$-dimensional vector space, however, it is productive as illustration to display the projections onto 2-dimensional subspaces. In [1], we have chosen 36 subspaces involving pairs of $M_{W}, R_{\nu}, C_{1+}($ iso $), A_{L R}(L E P), \sigma_{\nu} /\left(\sigma_{\nu}+\sigma_{\nu_{e}}\right), A_{F B}(b), A_{p o l}(\tau)$, $\Gamma_{1 i}$, and $\Gamma_{Z}$, which are representative of the most sensitive observables. The result is that it will be possible to distinguish among most of the examples of new physics considered here with reasonable confidence. The types of new physics which manifest themselves at the tree level all have distinctive patterns. These are different from the patterns induced by loop effects with the exception of non-standard Higgs fields. which cannot be distinguished from $h_{V}$ by the measurements treated here. 
Table 5.8: Summary of the discussion in section 5: implications for non-standard Higgs fields $(\lambda=$ $\left.\rho_{0}-1\right)$, leptoquarks $\left(\lambda=\frac{2^{1 / 2}|\eta|^{2}}{8 M_{S}^{2} G_{F}}\right)$, and four fermi operators $\left(\lambda=\frac{2^{1 / 2} \pi}{G_{F} \Lambda^{2}}\right)$. The smallest value $\lambda^{m i n}$ is the value of $\lambda$ at which these new physics are manifested in the corresponding most sensitive observable

\begin{tabular}{cccl}
\hline \hline New Physics & Smallest $\lambda^{\text {min }}$ & Implications & Most Sensitive Observables \\
\hline NS Higgs & 0.0015 & $V E V_{N H} \sim 7 \mathrm{GeV}$ & $R_{\nu,} A_{L R}^{(L E P)}, M_{W} ;$ \\
& & & $A_{L R}^{(S L C)}, A_{F B}(b), \Gamma_{l i}, \Gamma_{Z}$ \\
Leptoquark & 0.0005 & $\frac{M_{s}}{\eta_{L}} \sim 5.5 \mathrm{TeV}$ & $C_{1+(i s o) ; C_{+}, 2 C_{1 u}+C_{1 d}}$ \\
4-Fermi op. (I) & 0.0023 & $\Lambda_{1} \sim 12.9 \mathrm{TeV}$ & $C_{2 p}(1) ; R_{\nu}$ \\
4-Fermi op. (II) & 0.0032 & $\Lambda_{2} \sim 10.9 \mathrm{TeV}$ & $\sigma_{\nu} /\left(\sigma_{\bar{\nu}}+\sigma_{\nu}\right)$ \\
4-Fermi op. (III) & 0.0002 & $\Lambda_{3} \sim 44 \mathrm{TeV}$ & $C_{1+(}($ iso $)$ \\
\hline \hline
\end{tabular}

Distinguishing new loop effects $\left(h_{A}\right.$ and $\left.h_{V}\right)$ from $M_{H}$ and $m_{t}$, or even $M_{H}$ and $m_{t}$ from each other is more difficult, though not impossible. It is likely, however, that $m_{t}$ will be accurately known from direct observation at the Tevatron within a few years. It is also possible that the Higgs boson will be directly observed as well. With that information, one could certainly elicit $h_{A}$ and $h_{V}$. If $M_{H}$ is not known from direct observation its effects on the observables considered here are generally small, though non-negligible for $M_{H} \leq 1 \mathrm{TeV}$. Assuming the exact validity of the SM (i.e., $h_{A}=h_{V}=0$ ), precision measurements could yield an estimate of $M_{H}$ with a sensitivity of a few hundred $\mathrm{GeV}$.

\section{Conclusions}

Rased upon experimental improvements and the theoretical framework developed in [1], the implications of high precision electroweak experiments to new physics have been studied. The framework was set up by taking several steps: (i) Using $M_{Z}$ as input, the predictions of the SM for 28 additional observables encompassing weak neutral current phenomena and intermediate vector boson masses and decay widths were calculated numerically to the one-loop level. (ii) The modifications of each of these SM predictions which might be caused by 10 general types of possible new physics were calculated numerically for 27 specific examples. (iii) The latter values, each subject to the specification of a single coupling strength parameter, were compared with the projected numerical experimental errors of future high precision experiments with a three-fold purpose: testing the SM (including radiative corrections) at the highest level of precision, recognizing the relative sensitivities of the several observables to the different types of new physics treated here, and delineating the nature of the new physics if deviations from the SM are found empirically. The detailed numerical results of the analysis were presented in 27 tables and accompanying figures. 
Table 5.9: Summary of the discussion in section 5: implications for heavy physics through loops. For $h_{V}, \lambda=\alpha h_{V} ;$ for $h_{A}, \lambda=2^{1 / 2} G_{F} M_{Z}^{2} h_{A} / 4 \pi ;$ for the variations of $m_{t}$ and $M_{H}, r_{a}=\left|\Delta O_{a}\right| / \Delta O_{a}^{\exp }$. The smallest value $\lambda^{\mathrm{min}}$ is the value of $\lambda$ at which these new physics are manifested in the corresponding most sensitive observable.

\begin{tabular}{|c|c|c|c|}
\hline New Physics & Srnallest $\lambda^{\text {min }}$ & Implications & Most Sensitive Observabless \\
\hline$h_{V}$ & 0.0015 & $h_{V} \sim 0.21$ & $\begin{array}{l}R_{\nu}, A_{L R}^{(L E P)}, M_{W} ; \\
A_{L R}^{(S L C)}, A_{F B}^{(b)}, \Gamma_{l i}, \Gamma_{Z}\end{array}$ \\
\hline$h_{A}$ & 0.0017 & $h_{A} \sim 0.16$ & $\begin{array}{l}A_{L R}^{(L E P)} ; \\
A_{L R}^{(S L C)}, C_{1+}(i s o), A_{F B}(b)\end{array}$ \\
\hline$M_{H}=50 \mathrm{GeV}$ & - & $r_{a} \sim 1.75$ & $\begin{array}{l}A_{L R}^{(L E P)} ; \\
M_{W}, R_{\nu}, A_{L R}^{(S L C)}, A_{F B}(b)\end{array}$ \\
\hline$M_{H}=1000 \mathrm{GeV}$ & - & $r_{a} \sim 1.74$ & $\begin{array}{l}A_{L R}^{(L E P)} ; \\
M_{W}, R_{\nu}, A_{L R}^{(S L C)}, A_{F B}(b)\end{array}$ \\
\hline$m_{\mathrm{t}}=150 \mathrm{GeV}$ & - & $r_{a} \sim 3.2$ & $\begin{array}{l}R_{\nu}, A_{L R}^{(L E P)}, M_{W} \\
A_{L R}^{(S L C)}, g_{L}^{2}, \sigma_{\nu e} /\left(\sigma_{\nu e}+\sigma_{\nu e}\right) \\
\text { asymmetries } \\
2 C_{1 u}+C_{1 d}, \Gamma_{l \bar{l}}, \Gamma_{Z}\end{array}$ \\
\hline$m_{\imath}=200 \mathrm{GeV}$ & - & $r_{a} \sim 4.0$ & $\begin{array}{l}R_{\nu}, A_{L R}^{(L E P)}, M_{W} \\
\text { asymmetries } \\
A_{L R}^{(S L C)}, g_{L}^{2}, \sigma_{\nu e} /\left(\sigma_{\nu, e}+\sigma_{\nu e}\right), \\
2 C_{1 u}+C_{1 d}, \Gamma_{l i}, \Gamma_{Z}\end{array}$ \\
\hline
\end{tabular}

In this paper, we have changed the inputs for theoretical predictions $M_{Z}=$ $91.177 \rightarrow 91.187 \mathrm{GeV}, m_{t}=100 \rightarrow 150 \mathrm{GeV}$, and $M_{H}=100 \rightarrow 250 \mathrm{GeV}$. We have also incorporated other new experimental results in our analysis. The three general conclusions emerged from the original analysis are still intact. They are: First, an analytic framework is necessary for the understanding of future high precision experiments and their interpretation within the SM. As noted earlier, the results of most electroweak experiments depend on the value of the weak mixing angle, $\sin ^{2} \theta_{W}$. A single precision measurement usually just specifies that angle. To constrain the theory, the results of equivalent precision measurements of other observables are necessary. Furthermore, the sensitivity of different observables to $\sin ^{2} \theta_{W}$ and to the radiative corrections varies significantly so that comparison of the values of $\sin ^{2} \theta_{W}$ extracted from several experiments may not by itself provide the most stringent test of their internal consistency. In addition, precise numerical comparison of one experiment with another, or experiment with theory, requires the top quark mass and Higgs mass to be known or at least constrained within reasonably tight limits. All of these difficulties may be overcome with a sufficient number of high precision experiments analyzed within a global or general framework such as proposed in [1]. The absence of the framework will lead to a waste of the high precision of these experiments and limit the conclusions to be drawn from them.

Second, the outcome of the global analysis will include accurate determina- 
tions of the radiative corrections within the SM, which will test the gauge nature of the theory at its foundation. The high precision experiments uniquely verify renormalizibility of the theory and the consistency of the calculations of the radiative corrections.

Finally, the variety of observables open to precise experimental study and the precise nature of the electroweak theoretical predictions jointly constitute a powerful means of searching for new physics. The wide range of momentum transfers and the varied sensitivities of the electroweak phenomena to new physics form a network through which new physics in the $\mathrm{TeV}$ region is unlikely to pass unnoticed. We had tried to furnish examples of this by considering specific instances of possible new physics. In general, all of the measurements considered show good sensitivity to one or another type of the new physics treated. Possibly, the new physics nature holds in store for us is beyond our imagination right now, which offers still another reason to encourage the performance of a multiplicity of measurements. Precision measurements of the masses of $Z$ and $W$, precise determinations of neutrino-quark and neutrino-electron scattering cross-sections, precision measurements of the parameters of atomic parity-violation, and precision measurements of the asymmetries in $e^{+} e^{-}$scattering at the $Z$-pole, combined with precise knowledge of the $Z$-decay widths are all possible, and to a greater or lesser extent are possible with existing accelerators and, in many cases, with existing detectors.

This circumstance, global in character experimentally and theoretically, presents a window of opportunity through which a view of the future realm of elementary particle physics may be obtained before we are transported there by the forthcoming high energy colliders. We must use our resources wisely to take advantage of the opportunity.

One conspicuous change of the picture is the emerging importance of the $Z$ decay widths in our analysis, which following naturally from their better than anticipated precision. $\Gamma_{1 I}$ and $\Gamma_{Z}$ are especially important. This verifies the wisdom of the precaution stated in section 7 of [1] that "... we do not wish to underestimate the inventiveness and ingenuity of the experimentalists who will carry out the future electroweak experiments." That proves once again the validity of using high precision experiments to test the SM and to find new physics beyond. That also points out the importance of global analysis similar to this one.

\section{Acknowledgements}

It is appropriate to thank all my colleagues and friends in physics. Especially I am grateful to Paul G. Langacker, Alfred K. Mann, and William J. Marciano; it has been a great pleasure to know and to work with them.

\section{References}

1. P. Langacker, M. Luo, and A. Mann, Rev. of Mod. Phys. Vol. 64 (1992) 87. 
2. L. Rolandi, XXVI ICHEP, Aug. 1992.

3. P. Langacker, private communication.

4. Weinberg, S., Phys. Rev. Lett. 19 (1967) 1264; Salam, A., 1969, in Elementary Particle Theory, ed. N. Svartholm (Almquist and Wiksells, Stockholm, 1969) p. 367; Glashow, S.L., Iliopoulos, J., and Maiani, L., Phys. Rev. D2 (1970) 1285.

5. See, for example, P. Langacker, Structure of the Standard Model, this book.

6. D. Schaile, Z-Pole Experiments, this book.

7. A. Blondel, Polarization in $e^{+} e^{-}$annihilation, this book.

8. D. Treille, The LEP II Program, this book.

9. D. Burke, Future $e^{+} e^{-}$Colliders at High Energy, this book.

10. F. Perrier, The Measurement of Electroweak Parameters from Deep Inelastic Neutrino Scattering, this book.

11. A. K. Mann, Elastic Neutrino-Nucleon Scattering, this book.

12. J. Panman, Neutrino-Electron Scattering, this book.

13. C. Wieman, Atomic Parity Violation Experiments, this book.

14. W. Hollik, Renormalization of the Standard Model and Predictions for $e^{+} e^{-}$ Processes, this book.

15. W. Marciano, Radiative Corrections to Neutral Current Processes, this book.

16. J. Sapirstein, The Theory of Atomic Parity Violation, this book.

17. See [13]. The uncertainties in the $C s$ nuclear structure may cause extra theoretical errors. A rough estimate shows the effect is negligible, but further investigation is required to get a definite conclusion. See, for example, S. Pollock, E. Fortson, and L. Willets, preprint 40561-050-INT92-00-14, to be published in Phys. Rev. C.

18. D. London, Exotic Fermions, this book. Se also [1] and references within. 

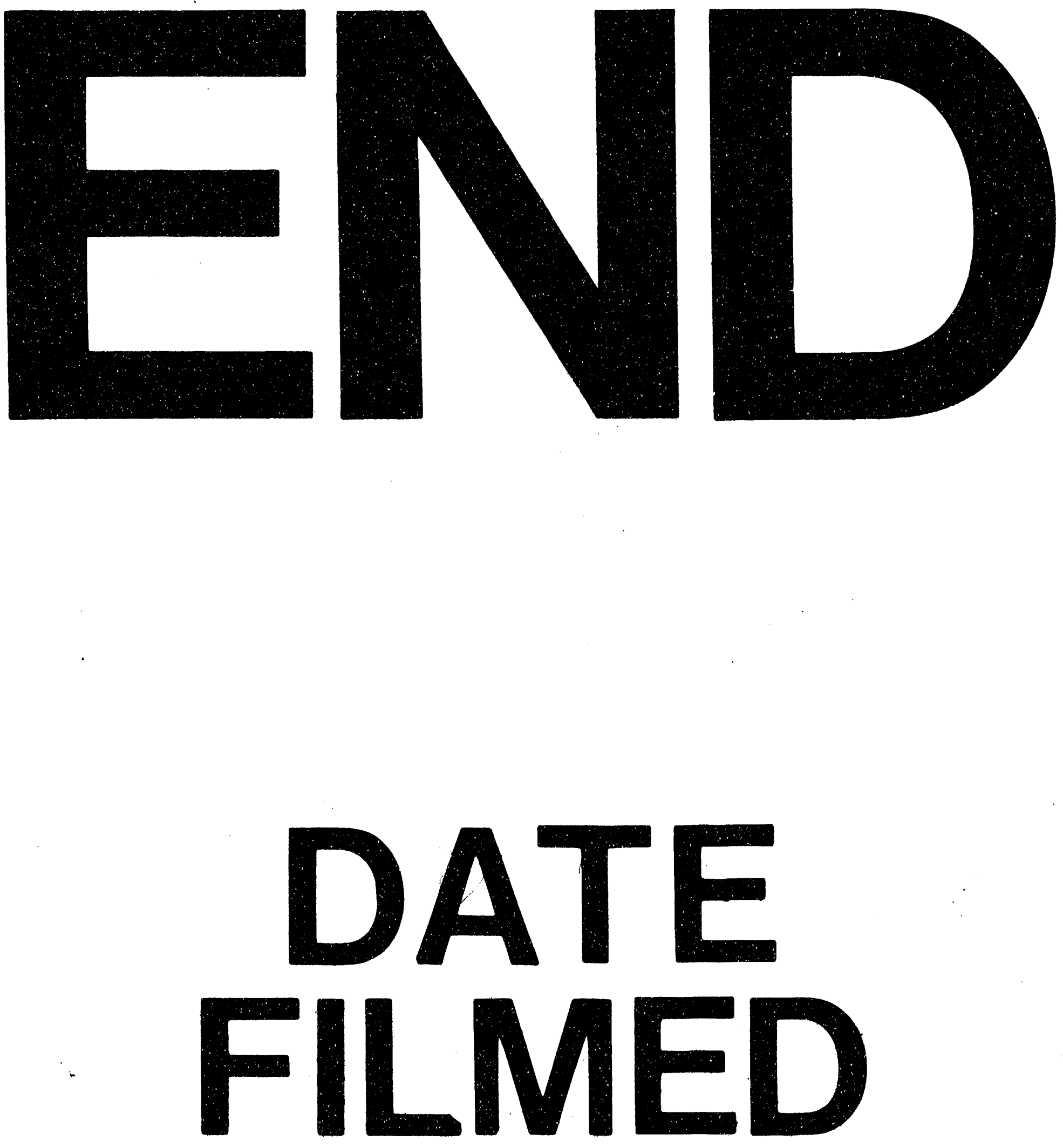

1

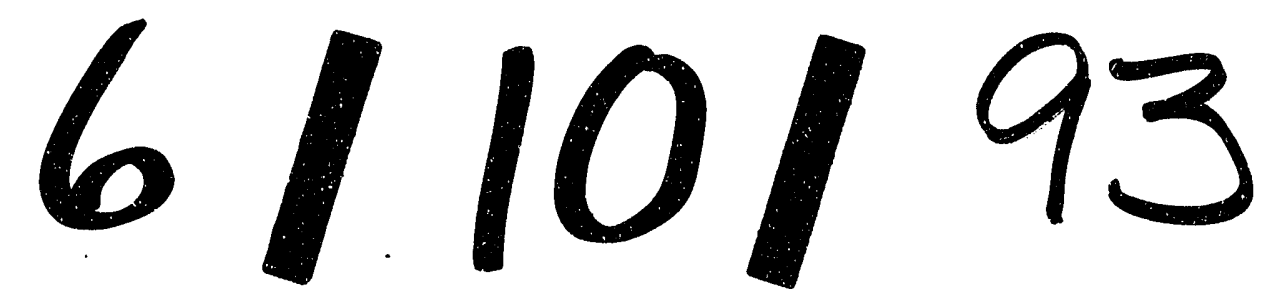


